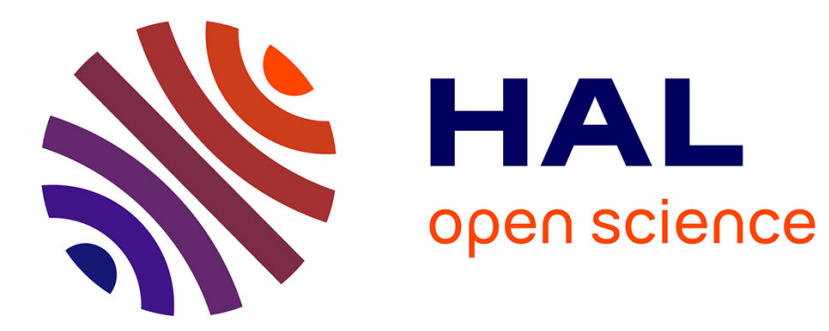

\title{
Exergy-based Aircraft Aeropropulsive Performance Assessment: CFD Application to Boundary Layer Ingestion
}

\author{
A. Arntz, O. Atinault, D. Destarac, A. Merlen
}

\section{To cite this version:}

A. Arntz, O. Atinault, D. Destarac, A. Merlen. Exergy-based Aircraft Aeropropulsive Performance Assessment: CFD Application to Boundary Layer Ingestion. AIAA AVIATION 2014, Jun 2014, ATLANTA, United States. hal-01068957

\section{HAL Id: hal-01068957 \\ https://hal-onera.archives-ouvertes.fr/hal-01068957}

Submitted on 26 Sep 2014

HAL is a multi-disciplinary open access archive for the deposit and dissemination of scientific research documents, whether they are published or not. The documents may come from teaching and research institutions in France or abroad, or from public or private research centers.
L'archive ouverte pluridisciplinaire HAL, est destinée au dépôt et à la diffusion de documents scientifiques de niveau recherche, publiés ou non, émanant des établissements d'enseignement et de recherche français ou étrangers, des laboratoires publics ou privés. 


\title{
Exergy-based Aircraft Aeropropulsive Performance Assessment: CFD Application to Boundary Layer Ingestion
}

\author{
Aurélien Arntz*, Olivier Atinault ${ }^{\dagger}$, Daniel Destarac ${ }^{\ddagger}$ and Alain Merlen $^{\S}$ \\ ONERA - The French Aerospace Lab
}

\begin{abstract}
Aircraft have evolved into extremely complex systems that require adapted tools to allow efficient design processes. A new formulation based on an exergy balance is under development at ONERA for assessing the aeropropulsive performance of future aircraft configurations. A control volume analysis is performed to relate the exergy supplied by the propulsion system, its partial destruction within the control volume and the aircraft mechanical equilibrium. The formulation does not rely on the expression of thrust and drag and is therefore especially suitable for the performance evaluation of aircraft configurations with boundary layer ingestion (BLI). A first step towards such applications is the investigation of a more academical configuration consisting in the ingestion by a powered nacelle of the complete wake of a simplified fuselage. Investigation is made via 3D RANS computations and it is shown that the benefit is due to lower levels of exergy destruction in the wake/jet of the BLI configuration.
\end{abstract}

\section{Introduction}

\section{I.A. Boundary Layer Ingestion}

For current civil aircraft configurations, the propulsion system is installed on the airframe via pylons to avoid interactions as much as possible. However there is a growing interest in the aerospace community in the application of the concept of boundary layer ingestion which typically consists in placing the engine on top of a blended-wing body configuration to ingest the boundary layer, see middle sketch of Fig. 1. It is derived from a more general concept, namely wake ingestion, which has long been known in the field of marine propulsion. ${ }^{25}$ The principle is to place the engine downstream of the body in order to ingest its wake and to ideally re-energize the wake by just the right amount to match freestream velocity, leaving no wake.

Considering the aircraft in steady flight, the deficit in momentum of the wake (blue area on Fig. 1) is balanced by the excess in momentum of the jet exhaust (green area). The basic benefit of ingesting the wake is twofold:

- In an airframe point of view, a first benefit can be explained via the kinetic energy associated with the wake and jet exhaust of a conventional aircraft configuration which are shown in orange areas in Fig. 1. The difference with ideal wake ingestion is determinant: there is no kinetic energy left in the wake. Mechanical energy analysis ${ }^{12}$ shows that there is a price to pay for any kinetic energy remaining in the wake and that its reduction, or ideally its cancellation, allows less energy to be supplied to the flow, and therefore a lower fuel consumption.

- In a propulsive perspective, for a given force, less power needs to be supplied to a flow that enters the engine with a lower velocity. As derived by Plas, ${ }^{19}$ we consider the force $F$ created by the engine:

$$
F=\dot{m}\left(V_{2}-V_{1}\right)=\dot{m} \Delta V
$$

*PhD Student, Department of Applied Aerodynamics, aurelien.arntz@onera.fr.

$\dagger$ Research Engineer, Department of Applied Aerodynamics, olivier.atinault@onera.fr.

‡Research Engineer, Department of Applied Aerodynamics, daniel.destarac@onera.fr.

$\S$ Scientific Director of the Fluid Mechanics and Energetics Branch, alain.merlen@onera.fr. 

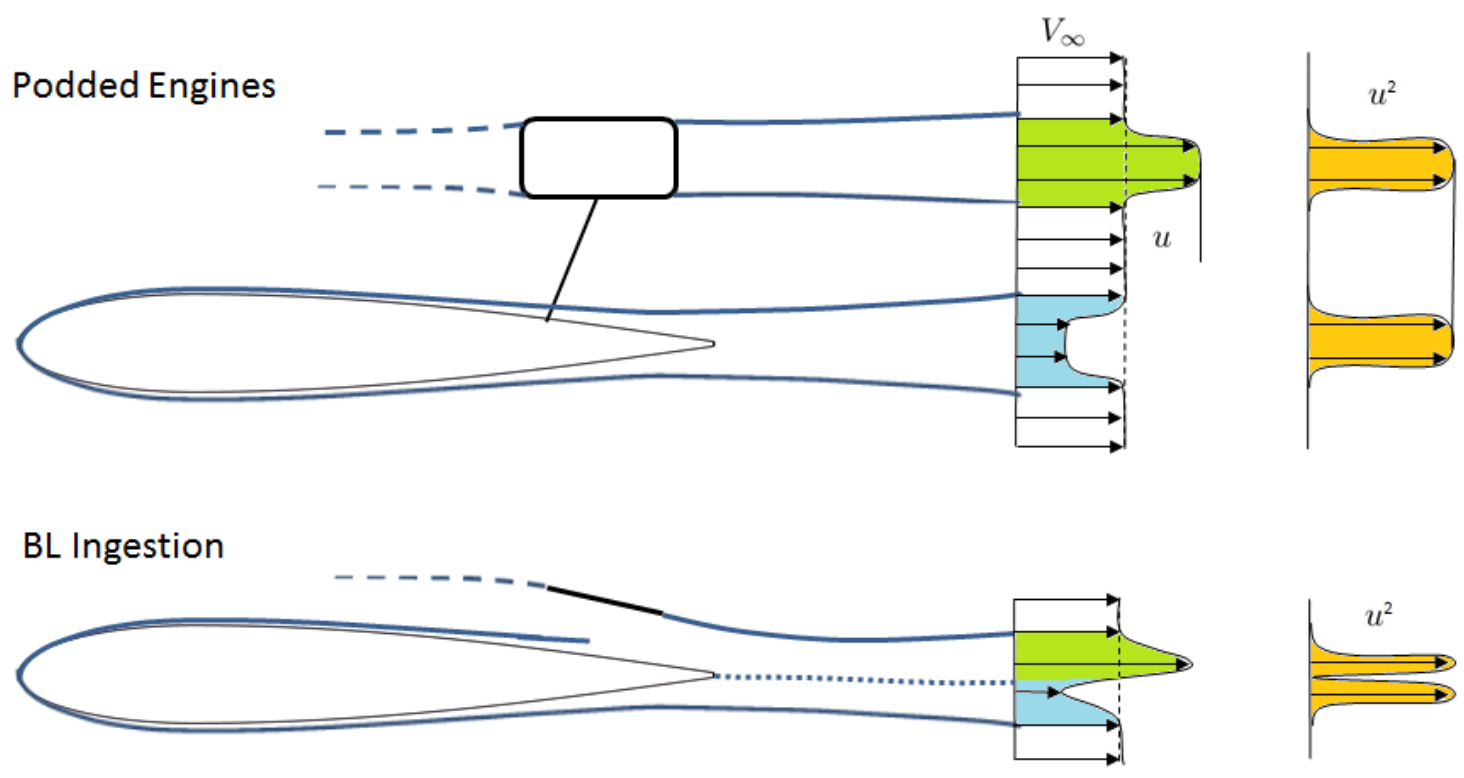

\section{Wake Ingestion}

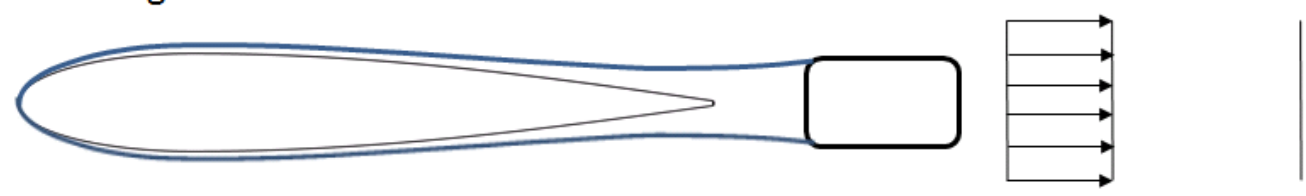

Figure 1. Wake ingestion concept: podded engine (top), boundary layer ingestion (middle) and ideal wake ingestion (bottom).

where $V_{1}$ and $V_{2}$ are respectively the inlet and outlet axial velocity and $\dot{m}$ is the massflow through the engine. The (mechanical) power $P$ supplied by the propulsion system is:

$$
P=\frac{\dot{m}}{2}\left(V_{2}^{2}-V_{1}^{2}\right)=F \frac{V_{1}+V_{2}}{2}=F\left(V_{1}+\frac{\Delta V}{2}\right)
$$

One can see that for constant massflow and constant force, $\Delta V$ is constant. Therefore, a decrease in $V_{1}$ results in a decrease in power supplied. A lower inlet velocity $V_{1}$ is typically experienced in the case of wake/boundary layer ingestion.

Studies on BLI have focused mainly on the performance of an unducted propeller ingesting a wake, as the theoretical considerations of Betz. ${ }^{7}$ Smith $^{25}$ introduced the power saving coefficient to quantify the benefit of the concept. Using an actuator disk to model the engine, he found examples exhibiting a benefit up to $20 \%$. Most previous studies have been made assuming incompressible flow and a static pressure equal to freestream static pressure at the inlet and outlet. This latter assumption is strong as it is known that close bodies generates aerodynamic interference (potential field effects ${ }^{25}$ ).

\section{I.B. Exergy-based Formulation}

The main motivation for deriving a new aerodynamic performance formulation is the BLI case described above for which conventional drag/thrust bookkeepings become inapplicable. The distinction between thrust and drag becomes ambiguous for such highly integrated propulsion systems. Drela ${ }^{12}$ recently proposed a formulation based on a mechanical energy analysis which does not rely on the expression of forces (thrust/drag). Among its applications could be cited the one of $\mathrm{Sato}^{23}$ for the optimization of hybrid wing-body configurations with BLI propulsion systems and the experimental and numerical assessment of the D8 aircraft. ${ }^{18,26}$

The formulation presented here takes into account the thermal energy in addition to the mechanical energy. These are the two forms of energy of a flowing fluid, forming the total enthalpy. In the perspective 
of producing work, any form of energy can be split in two: a first part theoretically fully convertible into mechanical work and a second part that is theoretically impossible to convert into mechanical work. Following the work of Rant ${ }^{20}$ to establish an international terminology, it appears that the words exergy ${ }^{\mathrm{a}}$ and anergy are well suited to represent these two forms. As exergy is the part of energy that has economic value, ${ }^{11}$ it is the part worth managing carefully. ${ }^{5}$

A detailed history of exergy can be found in Sciubba and Wall ${ }^{24}$ including theoretical aspects and engineering applications. Among recent airframe applications could be cited the investigation of entropy generation around the B747-200 by Alabi et. al. ${ }^{2}$ as well as the work of $\mathrm{Li}^{16}$ focusing on flows around 2D-airfoil and 3D-wing geometries. Regarding propulsion, most noticeable is the work of Roth ${ }^{22}$ to derive relationships between classic measures of component efficiencies to modern measures of work potential which have the advantage of being directly comparable to one another.

The present paper follows the recent effort of Riggins ${ }^{21}$ to bring together the equations of motion and an exergy balance.

\section{Theoretical Formulation}

The formulation sets a relationship between the aircraft mechanical (static) equilibrium and its exergy consumption. It consists in bringing together the equations of motion of the aircraft and a fluid flow analysis based on the first and second laws of thermodynamics. After having described the control volume in which the analysis is made, mass and momentum relations are applied to get a far-field expression for the power developed by the net force acting on the aircraft. This term will be later identified in an exergy balance made possible by the preliminary description of energy and anergy relations. The final exergy balance relates the exergy supplied by the propulsion system, its (partial) destruction within the control volume along with the aircraft mechanical equilibrium.

Note that the present formulation is restricted to steady flows.

\section{II.A. Preliminary Considerations}

We consider the aircraft as a mass moving at velocity $V_{\infty}$ and assume that the thrust $\mathrm{T}$ and the drag $\mathrm{D}$ act in the flight path direction, the resultant being $F_{x}$. A popular result is that a general power balance yields:

$$
(\mathrm{T}-\mathrm{D}) V_{\infty}=F_{x} V_{\infty}=W \dot{\Gamma}
$$

Where $W$ is the aircraft weight and $\Gamma$, defined as the energy height, is the sum of its potential and kinetic energies $^{\mathrm{b}}$. Eq. (3) means that any excess power $(\mathrm{T}-\mathrm{D}) V_{\infty}$ represents a potential for vertical and/or longitudinal accelerations, i.e. a gain in height and/or velocity. If $F_{x}=0$, the propulsion system provides as much thrust as the drag of the configuration and the aircraft is in mechanical equilibrium ${ }^{\mathrm{c}}, W \dot{\Gamma}=0$ and there is no potential for acceleration. Correspondingly, if the propulsion system provides more thrust than the drag of the configuration, the aircraft stores mechanical energy in the form of kinetic or potential energy.

A far-field expression of $W \dot{\Gamma}$ is found in the following paragraphs and is used subsequently in the exergy balance.

\section{II.B. Control Volume}

The fluid flow analysis is carried out in a continuous volume $\mathcal{V}$ delimited by the aircraft surface $\mathcal{S}_{A}$ and an outer boundary $\mathcal{S}_{O}$. Both surfaces are closed and a vector pointing outwards of the volume and locally normal to the surface is noted $\mathbf{n}$, as illustrated in Fig. 2.

The control volume delimits the system under study which is said to be thermodynamically open as it can exchange mass, work, and heat with the surrounding flow across its boundaries. The analysis is made in the aircraft reference frame, the control volume is therefore fixed and the air is flowing in and out of it.

\footnotetext{
${ }^{a}$ Other names can be found in the literature, especially availability made popular by Keenan. ${ }^{14}$

${ }^{\mathrm{b}} \dot{\Gamma}$ is the time rate of change of energy height.

${ }^{\mathrm{c}}$ We consider that all forces have the same point of application, that point being the aircraft center of gravity $G$, so that there is no torque imbalance.
} 


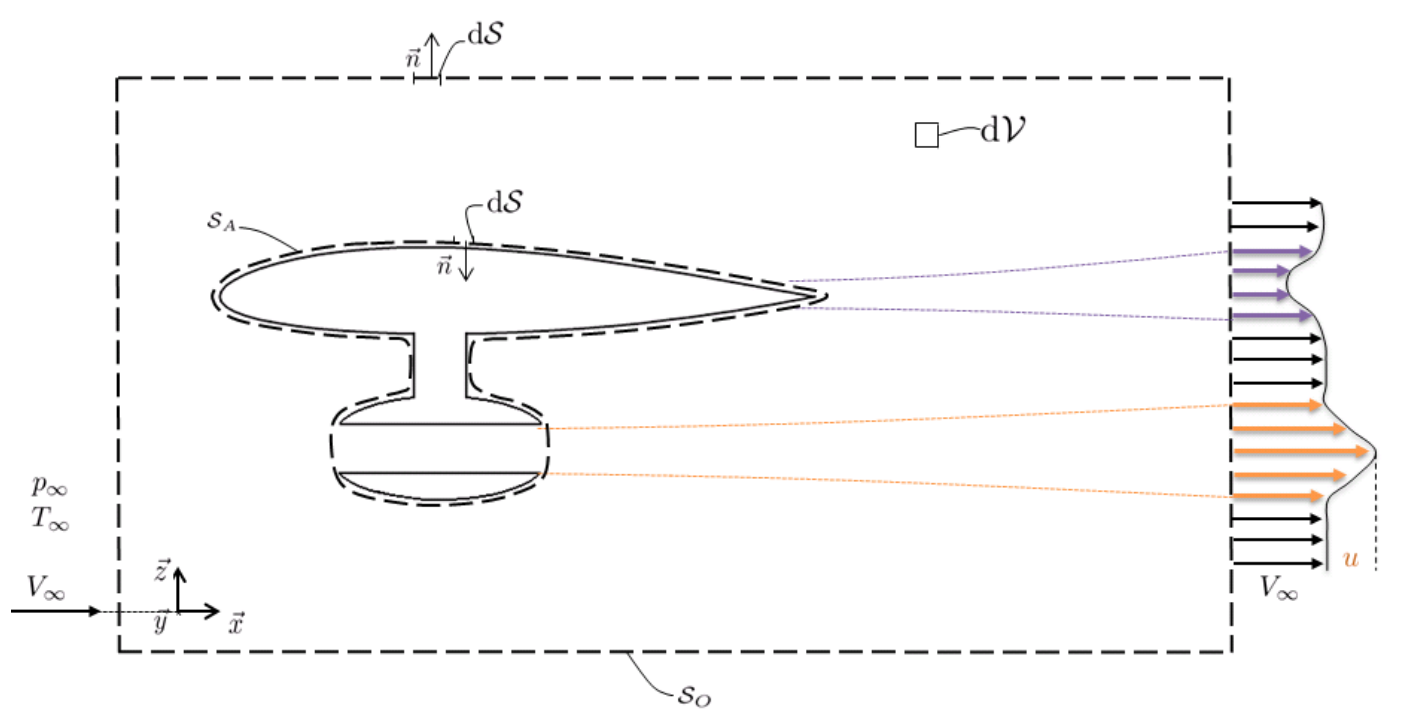

Figure 2. 2D cutaway view of $3 \mathrm{D}$ control volume surrounding the aircraft.

\section{II.C. Mass \& Momentum Relations}

\section{II.C.1. Mass Conservation}

The time-averaged mass relation in local form is as follows:

$$
\nabla \cdot(\rho \mathbf{V})=0
$$

Integrating within the control volume $\mathcal{V}$ and using the divergence theorem yields:

$$
\dot{m}_{A}=\dot{m}_{O}
$$

where:

$$
\begin{gathered}
\dot{m}_{A}:=-\int_{\mathcal{S}_{A}} \rho(\mathbf{V} \cdot \mathbf{n}) \mathrm{d} \mathcal{S}=\dot{m}_{f} \approx 0 \\
\dot{m}_{O}:=\int_{\mathcal{S}_{O}} \rho(\mathbf{V} \cdot \mathbf{n}) \mathrm{d} \mathcal{S}=\dot{m}_{f} \approx 0
\end{gathered}
$$

The fuel mass flow $\dot{m}_{f}$ will be assumed negligible compared to the air mass flow considered within the control volume, so that both integrals are zero, yielding for any scalar quantity convected by the flow like the total enthalpy $h_{i}$ :

$$
\int_{\mathcal{S}_{O}} \rho h_{i}(\mathbf{V} \cdot \mathbf{n}) \mathrm{d} \mathcal{S}=\int_{\mathcal{S}_{O}} \rho\left(h_{i}-h_{i_{\infty}}\right)(\mathbf{V} \cdot \mathbf{n}) \mathrm{d} \mathcal{S}=\int_{\mathcal{S}_{O}} \rho \delta h_{i}(\mathbf{V} \cdot \mathbf{n}) \mathrm{d} \mathcal{S}
$$

This property is used later in the development process.

\section{II.C.2. Momentum Relation}

The time-averaged momentum relation in integral form is as follows:

$$
\nabla \cdot(\rho \mathbf{V} \otimes \mathbf{V})=-\nabla \mathbf{p}+\nabla \cdot \overline{\bar{\tau}}
$$

Again, integrating within the control volume and invoking the divergence theorem yields the two following dynalpy fluxes:

$$
\mathbf{F}_{\mathrm{A}}=\mathbf{F}_{\mathbf{O}}
$$

$$
4 \text { of } 21
$$


where $\mathbf{F}_{\mathbf{A}}$ is the net force acting on the aircraft and $\mathbf{F}_{\mathbf{O}}$ is a far-field expression of the same force:

$$
\begin{gathered}
\mathbf{F}_{\mathbf{A}}:=\int_{\mathcal{S}_{A}}\left[\rho \mathbf{V}(\mathbf{V} \cdot \mathbf{n})+\left(p-p_{\infty}\right) \mathbf{n}-(\overline{\bar{\tau}} \cdot \mathbf{n})\right] \mathrm{d} \mathcal{S} \\
\mathbf{F}_{\mathbf{O}}:=-\int_{\mathcal{S}_{O}}\left[\rho \mathbf{V}(\mathbf{V} \cdot \mathbf{n})+\left(p-p_{\infty}\right) \mathbf{n}\right] \mathrm{d} \mathcal{S}
\end{gathered}
$$

Note that the viscous force has been dropped from (10b) as it is rapidly negligible when the outer boundary $S_{O}$ is moved away from the body ${ }^{\mathrm{d}}$ compared to the other forces involved. As mentioned in the previous paragraph, the rate of work done by this force is $W \dot{\Gamma}$ :

$$
W \dot{\Gamma}=\int_{\mathcal{S}_{O}}\left[\rho u V_{\infty}(\mathbf{V} \cdot \mathbf{n})+\left(p-p_{\infty}\right)\left(\mathbf{V}_{\infty} \cdot \mathbf{n}\right)\right] \mathrm{d} \mathcal{S}
$$

Note that this expression represents the power of the resultant force and that there is no need to distinguish thrust from drag. The following sections aims at building a first and second law balance which will contain this far-field expression of $W \dot{\Gamma}$.

\section{II.D. Exergy Analysis}

\section{II.D.1. Definitions and Terminology}

Exergy is a thermodynamic property describing the maximum theoretical work that can be obtained from a substance in taking it from a given temperature and pressure/velocity to a state of thermal and mechanical equilibrium with the environment. It is a state property in that it is a function of both the state of the system and of the state of the surroundings. The latter is here taken as the atmosphere at the altitude of flight, whose properties are noted with the subscript $\infty$.

As mentioned in the introduction, we can say that exergy = energy - anergy, which can be translated mathematically into:

$$
\varepsilon=\left(h_{i}-h_{i_{\infty}}\right)-T_{\infty}\left(\mathrm{s}-\mathrm{s}_{\infty}\right)
$$

where we have neglected the gravitational potential energy of the specific flow exergy ${ }^{6}$. We are interested in calculating the time-averaged change in exergy which can be written as:

$$
\nabla \cdot(\rho \varepsilon \mathbf{V})=\nabla \cdot\left(\rho \delta h_{i} \mathbf{V}\right)-T_{\infty} \nabla \cdot(\rho \delta \mathbf{s} \mathbf{V})
$$

The two following sections aim at finding an expression for the two right-hand side terms of Eq. (13).

\section{II.D.2. Energy}

The total enthalpy is defined as the sum of the (static) enthalpy and of the kinetic energy. It can therefore be expanded as $\delta h_{i}=\delta e+\delta(p / \rho)+\delta V^{2} / 2$, where $\delta e$ is the relative internal energy. The (time-averaged) change in total enthalpy can be written as:

$$
\nabla \cdot\left(\rho \delta h_{i} \mathbf{V}\right)=\nabla \cdot(\rho \delta e \mathbf{V})+p_{\infty} \nabla \cdot \mathbf{V}+\nabla \cdot\left(p-p_{\infty}\right) \mathbf{V}+\nabla \cdot\left(\rho \frac{V^{2}}{2} \mathbf{V}\right)
$$

where we have added and subtracted $p_{\infty} \nabla \cdot \mathbf{V}$. This relation is used in section II.E.

\section{II.D.3. Anergy}

The so-called Gibbs relation provides a relation between entropy s and internal energy $e$ :

$$
T \mathrm{ds}=\mathrm{d} e+p \mathrm{~d} \frac{1}{\rho}
$$

\footnotetext{
${ }^{\mathrm{d}} \mathrm{A}$ few chords downstream of the configuration, see Oswatitsch. ${ }^{17}$
} 
Inserting the expression of the internal energy variation into the Gibbs relation yields the following (time-averaged) entropy relation:

$$
T \nabla \cdot(\rho \delta \mathbf{s} \mathbf{V})=(\overline{\bar{\tau}} \cdot \nabla) \cdot \mathbf{V}-\nabla \cdot \mathbf{q}
$$

And introducing the Fourier law $\mathbf{q}=-k \nabla \mathbf{T}$, where $k$ is the thermal conductivity of the air, and the viscous dissipation $\Phi=(\overline{\bar{\tau}} \cdot \nabla) \cdot \mathbf{V}$, one gets:

$$
\begin{aligned}
\nabla \cdot(\rho \delta \mathrm{s} \mathbf{V}) & =\frac{\Phi}{T}-\frac{1}{T} \nabla \cdot \mathbf{q} \\
& =\frac{\Phi}{T}+\frac{k}{T^{2}}(\nabla \mathbf{T})^{2}-\nabla \cdot \frac{\mathbf{q}}{T}
\end{aligned}
$$

Multiplying Eq. (17) by the reference temperature $T_{\infty}$ yields the following anergy relation:

$$
T_{\infty} \nabla \cdot(\rho \delta \mathbf{s} \mathbf{V})=\frac{T_{\infty}}{T} \Phi+\frac{T_{\infty}}{T^{2}} k(\nabla \mathbf{T})^{2}-\nabla \cdot \frac{T_{\infty}}{T} \mathbf{q}
$$

This relation is used in the following section.

\section{II.E. Exergy Balance}

To simplify the derivation we neglect the heat transfer across the outer boundary as well as viscous terms ${ }^{\mathrm{e}}$. From the divergence theorem, the integration within the control volume of Eq. (13) yields:

$$
-\int_{\mathcal{S}_{A}} \rho \varepsilon(\mathbf{V} \cdot \mathbf{n}) \mathrm{d} \mathcal{S}=-\int_{\mathcal{V}} \nabla \cdot \rho \varepsilon \mathbf{V} \mathrm{d} \mathcal{V}+\int_{\mathcal{S}_{O}} \rho \varepsilon(\mathbf{V} \cdot \mathbf{n}) \mathrm{d} \mathcal{S}
$$

which, inserting Eqs. (14 \& 18) and using Eqs. (7 \& 11), yields the following exergy balance:

$$
\dot{\mathcal{E}}_{\text {prop }}=W \dot{\Gamma}+\dot{\mathcal{E}}_{m}+\dot{\mathcal{E}}_{t h}+\dot{\mathcal{A}}_{\mathcal{V}}
$$

All terms are defined hereafter and sketched in Fig. 3.

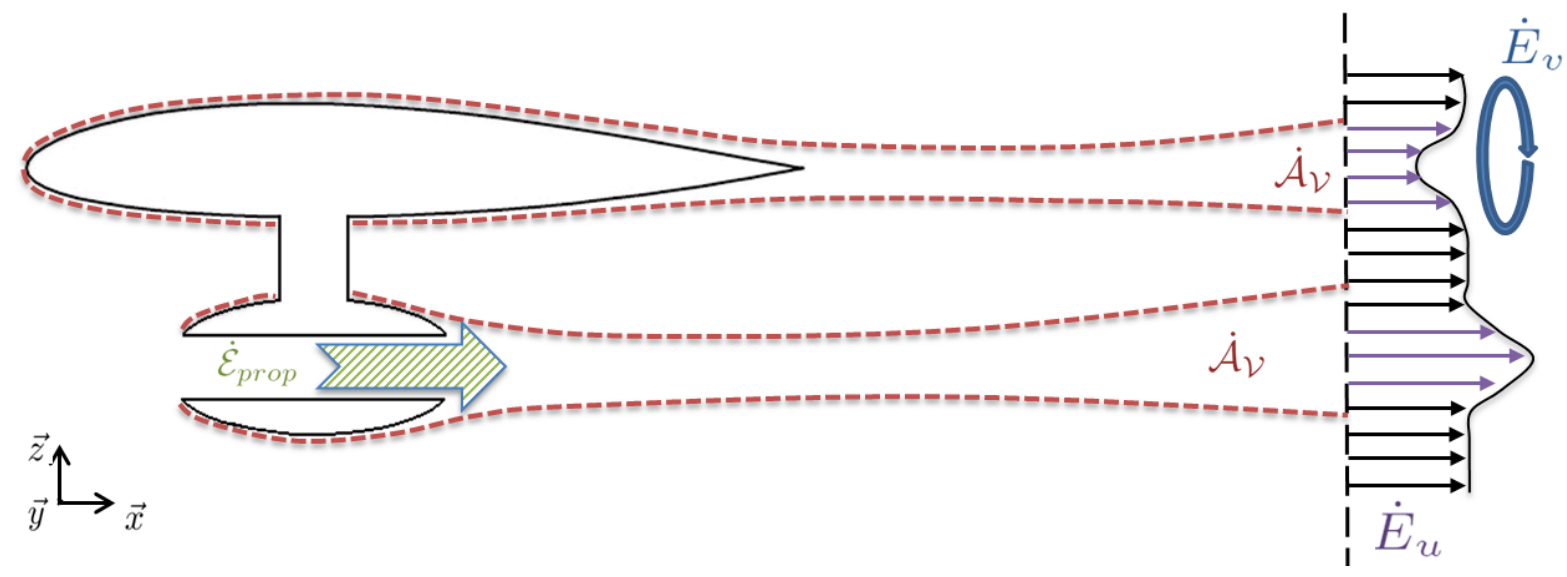

Figure 3. Main terms of the exergy balance in the flow field, $\dot{\mathcal{E}}_{t h}$ and $\dot{E}_{p}$ are not shown.

$\dot{\mathcal{E}}_{\text {prop }}$ : the exergy supplied by the propulsion system ${ }^{\mathrm{f}}$

$$
\dot{\mathcal{E}}_{\text {prop }}:=-[\underbrace{\int_{\mathcal{S}_{P}} \rho \delta h_{i}(\mathbf{V} \cdot \mathbf{n}) \mathrm{d} \mathcal{S}}_{\text {Total Power }}-\underbrace{T_{\infty} \int_{\mathcal{S}_{P}} \rho \delta \mathbf{s}(\mathbf{V} \cdot \mathbf{n}) \mathrm{d} \mathcal{S}}_{\text {Propulsive Anergy }}]
$$

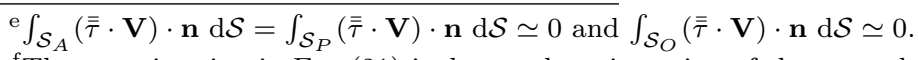

${ }^{\mathrm{f}}$ The negative sign in Eq. (21) is due to the orientation of the normal vector $\mathbf{n}$; it ensures $\dot{\mathcal{E}}_{\text {prop }} \geq 0$. 
Both terms are non-zero only on permeable surfaces of the aircraft surface $S_{A}$ (where $\mathbf{V} \cdot \mathbf{n} \neq 0$ ). In the present analysis, this surface is considered to be only the one associated with the propulsion system $S_{P}$. The first term is the total power supplied to the flow and is therefore equal to the amount of energy released by the combustion of fuel. ${ }^{13} \mathrm{~A}$ decomposition of this term is provided in the following section. The second term represents all losses that have been produced within the propulsion system delimited by the surface $S_{P}$. The combination of both forms the exergy delivered by the propulsion system to the fluid.

$\dot{\mathcal{E}}_{m}$ : the outflow of mechanical exergy

$$
\dot{\mathcal{E}}_{m}:=\underbrace{\int_{\mathcal{S}_{O}} \frac{1}{2} \rho u^{2}(\mathbf{V} \cdot \mathbf{n}) \mathrm{d} \mathcal{S}}_{\dot{E}_{u}}+\underbrace{\int_{\mathcal{S}_{O}} \frac{1}{2} \rho\left(v^{2}+w^{2}\right)(\mathbf{V} \cdot \mathbf{n}) \mathrm{d} \mathcal{S}}_{\dot{E}_{v}}+\underbrace{\int_{\mathcal{S}_{O}}\left(p-p_{\infty}\right)\left[\left(\mathbf{V}-\mathbf{V}_{\infty}\right) \cdot \mathbf{n}\right] \mathrm{d} \mathcal{S}}_{\dot{E}_{p}}
$$

This is the sum of the longitudinal $\left(\dot{E}_{u}\right)$ and transverse $\left(\dot{E}_{v}\right)$ kinetic energies deposition rates and of the pressure-work rate $\left(\dot{E}_{p}\right)$, see Drela. ${ }^{12}$ As these terms are of mechanical nature, they represent exergy, and therefore indicate a potential for improvement.

As mentioned, in the introduction, $\dot{E}_{u}$ is typically associated with wakes/jet exhausts and is used to explain the benefit of BLI for the airframe. $\dot{E}_{v}$ is generally associated with (lift-induced) vortices, and can be valued through formation flight ${ }^{15}$ or wingtip turbines. ${ }^{1}$

For most aerospace applications, these terms are non-valued and therefore represent an exergy loss.

$\dot{\mathcal{E}}_{t h}:$ the outflow of thermal exergy

$$
\dot{\mathcal{E}}_{t h}:=\underbrace{\int_{\mathcal{S}_{O}} \rho \delta e(\mathbf{V} \cdot \mathbf{n}) \mathrm{d} \mathcal{S}}_{\dot{E}_{t h}}+\underbrace{\int_{\mathcal{S}_{O}} p_{\infty}(\mathbf{V} \cdot \mathbf{n}) \mathrm{d} \mathcal{S}}_{\dot{E}_{W}}-\underbrace{T_{\infty} \int_{\mathcal{S}_{O}} \rho \delta \mathbf{s}(\mathbf{V} \cdot \mathbf{n}) \mathrm{d} \mathcal{S}}_{\dot{\mathcal{A}}}
$$

Assuming a perfect gas, we have that internal energy is solely proportional to temperature, so that the first term is the thermal energy outflow. The second term is usually referred to as surrounding work in the literature. ${ }^{9}$ It corresponds to the boundary work done by the system to overcome the reference pressure field at $p_{\infty}$. The last term is the outflow of anergy.

Summing the three terms yields the maximum amount of work that is theoretically extractable from the thermal energy. To extract this work one should think about a reversible Carnot cycle using the thermal energy as a source.

For most aerospace applications, this term is non-valued and therefore represents an exergy loss.

$\dot{\mathcal{A}}_{\mathcal{V}}:$ the generation of anergy

$$
\dot{\mathcal{A}}_{\mathcal{V}}:=\underbrace{\int_{\mathcal{V}} \frac{T_{\infty}}{T} \Phi \mathrm{d} \mathcal{V}}_{\dot{\mathcal{A}}_{\phi}}+\underbrace{\int_{\mathcal{V}} \frac{T_{\infty}}{T^{2}} k(\nabla T)^{2} \mathrm{~d} \mathcal{V}}_{\dot{\mathcal{A}}_{\nabla T}}
$$

$\dot{\mathcal{A}}_{\phi}$ is linked to viscous dissipation which transforms any difference in kinetic energy into thermal energy. Therefore, this phenomenon acts towards reducing the mechanical energy to zero by building a new homogeneous field of velocity and pressure, in which the system and its surroundings are back in mechanical equilibrium. The viscous dissipation is a function of the square of the velocity gradients so that avoiding strong velocity changes would tend to reduce this term.

$\dot{\mathcal{A}}_{\nabla T}$ is linked to thermal mixing which reduces the difference in temperature therefore acting towards building a new homogeneous field of temperature, in which the system and its surroundings are back in thermal equilibrium. The thermal mixing is a function of the square of the temperature gradients so that avoiding strong temperatures variations would tend to reduce this term.

Both terms are strictly positive and hence will always increase anergy, they indicate irreversible processes that destroy exergy. For most civil aerospace applications, the anergy associated with viscous dissipation is the largest contributor to the overall anergy generation. 
As indicated by Drela, ${ }^{12} W \dot{\Gamma}$ becomes a power source in descent. Because the left-hand side term of Eq. (20) is a near-field term, the sum of all right-hand side terms has to be constant while the outer boundary is pushed far downstream of the configuration. Linearizing the Gibbs equation ${ }^{\mathrm{g}}$ demonstrates that the thermal exergy vanishes far downstream while it is admitted that all three terms of $\dot{\mathcal{E}}_{m}$ reduce to zero, ${ }^{12}$ due to viscous dissipation. As a consequence, one can see, assuming an aircraft at equilibrium, that all the exergy of the fuel (delivered by the propulsion system) is ultimately destroyed and that there is a one-to-one relation with the overall anergy generated.

In the absence of propulsion $\dot{\mathcal{E}}_{\text {prop }}=0$ and $W \dot{\Gamma}$ reduces to the rate of work done by the net force acting on the configuration, namely drag. As a consequence, Eq. (20) reduces to:

$$
D V_{\infty}=\dot{\mathcal{E}}_{m}+\dot{\mathcal{E}}_{t h}+\dot{\mathcal{A}}_{\phi}+\dot{\mathcal{A}}_{\nabla T}
$$

In a thermodynamic point of view, the wake of the body is directly linked to the drag that it experiences. One can see that the reduction, or eventually the cancellation, of one of the right-hand side term could reduce the drag of the body.

Also note that all terms of the formulation can be expressed in power counts, abbreviated $p c$, in a direct analogy to the (more common) drag counts, abbreviated $d c$ :

$$
C \dot{E}_{u}:=\frac{\dot{E}_{u}}{q_{\infty} V_{\infty} A_{r e f}}
$$

By doing so, power terms are non-dimensionalized by the power of the dynamic pressure of the freestream $q_{\infty}$ and their magnitude are directly comparable to the (force) coefficients expressed in drag counts.

\section{Numerical Application}

The aim of this numerical application is to demonstrate the benefit of the formulation for a physical flow analysis. It is also a first step towards the investigation of realistic boundary layer ingestion propulsion systems placed on top of a blended-wing body.

\section{III.A. Test Case Presentation}

\section{III.A.1. Background}

The present study follows recent numerical and experimental aerodynamic investigations of BLI at Onera, see Atinault. ${ }^{4}$ The test case has been developed for validating both numerical simulations and experimental methodologies that will be necessary for the design of a future transport aircraft incorporating a BLI propulsive system. It consists in a rather academic configuration made up of a simplified fuselage geometry and a nacelle enclosing a fan. The fuselage has been designed to produce a wake whose diameter matches the one of the engine inlet, so that a $100 \%$ wake ingestion is achievable, see Fig. 4.
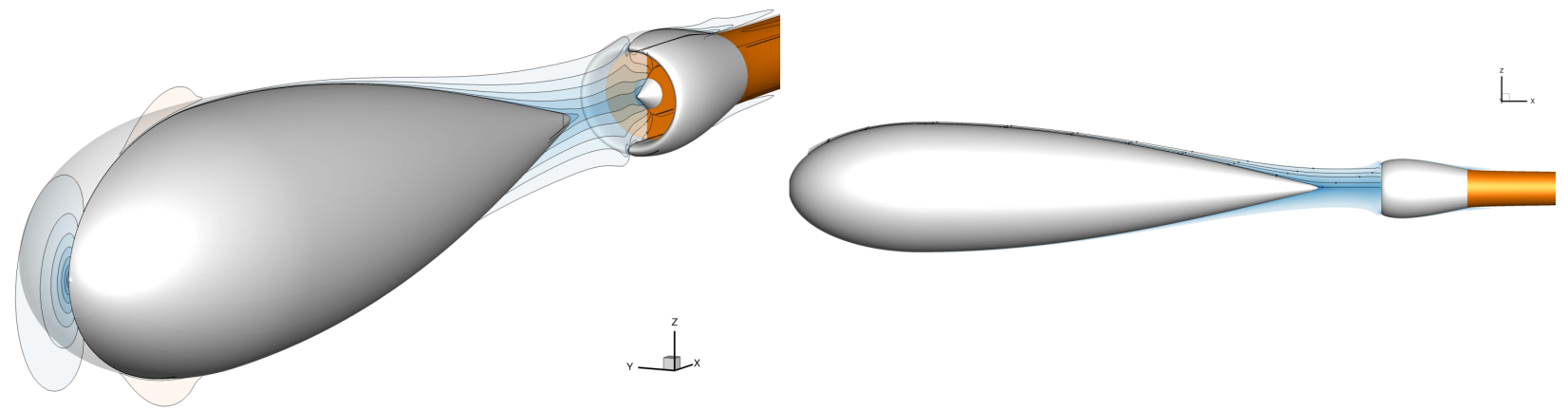

(a) 3D view.

(b) XZ-Plane view.

Figure 4. 3D representation of the BLI configuration ingesting the fuselage wake.

\footnotetext{
${ }^{\text {g Assuming }} p \approx p_{\infty}$ and $T \approx T_{\infty}$ we can linearize Eq. (15) around $\left(p_{\infty}, T_{\infty}\right)$ which directly leads a zero thermal exergy.
} 
The Reynolds number is set at $R e=4.5810^{6}$ based on a unit reference length. No incidence is considered and a low-speed Mach number of $M_{\infty}=0.2$ is set, so that the flow is in the low-compressible range.

As the flow is only weakly affected by thermal phenomena, if we assume that locally $T \approx T_{\infty}$, the thermal exergy becomes negligible and the anergy associated with thermal mixing vanishes. Also, the temperature ratio in front of the term associated with viscous dissipation becomes equal to one meaning that the exergybased formulation reduces to mechanical energy balance proposed by Drela. ${ }^{12}$

Two configurations will be compared to determine the benefit of the BLI arrangement:

- A BLI configuration which corresponds to the case where the nacelle is placed at a given distance downstream of the fuselage $(\Delta x=0.20$ for a fuselage length of 1.50). The aim being for the nacelle to ingest the entire wake of the fuselage, see Fig. 5. This configuration will be referred to as BLI.

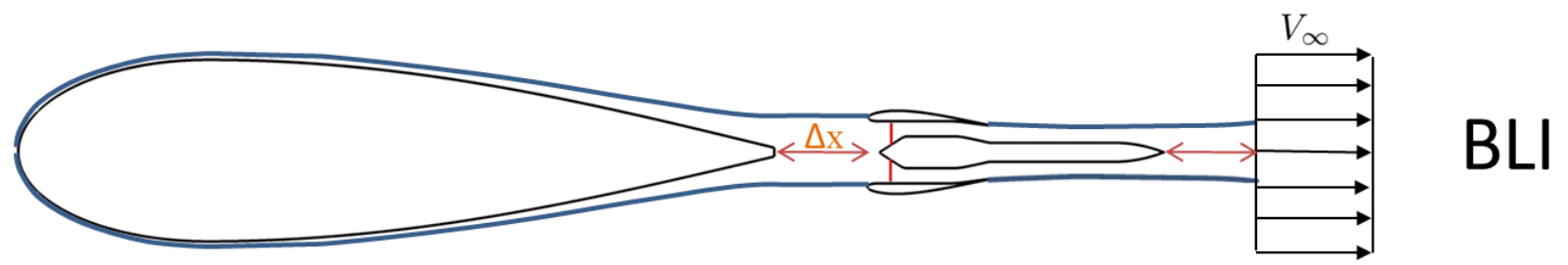

Figure 5. Simplified representation of the ideal BLI configuration being when the two bodies are close (therefore aerodynamically interacting) and when no wake is left downstream of the entire configuration.

Strictly speaking it is more a wake ingestion rather than a boundary layer ingestion, but the acronym BLI is used for convenience.

- A reference configuration which corresponds to the case where the fuselage and the nacelle are assumed to be sufficiently far from each other so that we can neglect any interaction between the two solids, see Fig. 6. This configuration represents a typical commercial aircraft architecture and will be referred to as REF.

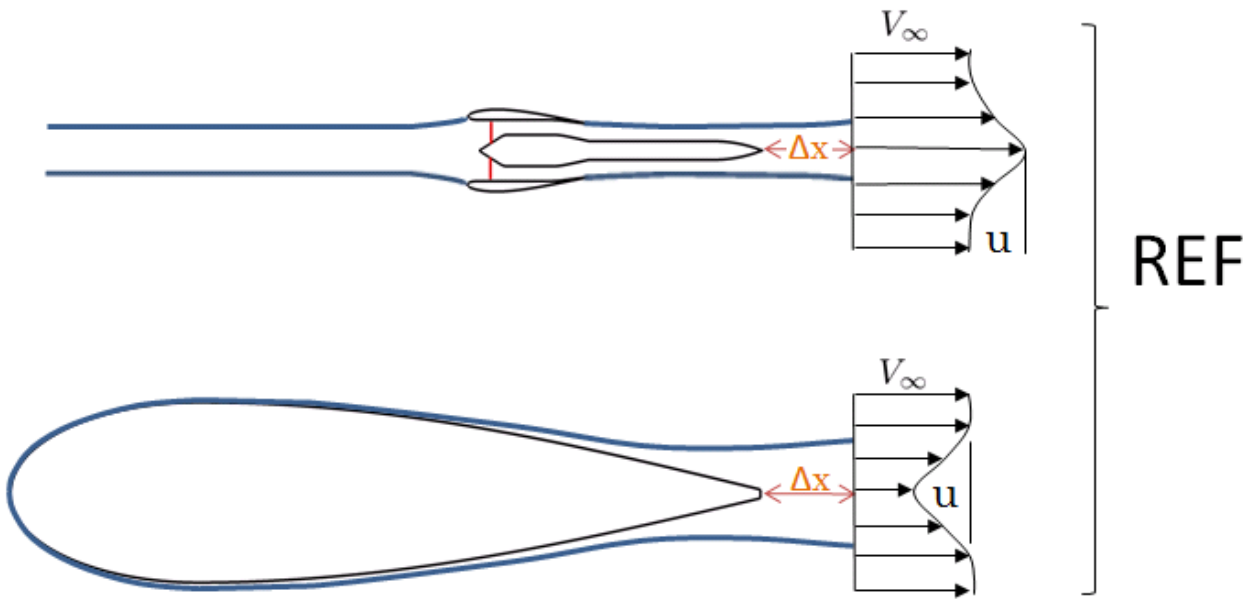

Figure 6. Simplified representation of the reference configuration being the combination of the isolated nacelle (top) and of the isolated fuselage (bottom).

\section{III.A.2. Figures of Merit}

Figures of merit (FoM) are required to quantify the benefit of the BLI arrangement. In the present analysis, all following FoM are calculated for fixed geometries and for a given net force acting on the entire configuration. 
Exergy-Saving Coefficient To quantify the BLI benefit, it is worth comparing the exergy saving from the initial usage. The adapted figure is therefore the exergy-saving coefficient:

$$
E S C:=\frac{\dot{\mathcal{E}}_{\text {prop }}^{R E F}-\dot{\mathcal{E}}_{\text {prop }}^{B L I}}{\dot{\mathcal{E}}_{\text {prop }}^{R E F}}=\frac{\text { Exergy Saving }}{\text { Reference Propulsive Exergy }}
$$

This is the most important FoM as it directly indicates how much exergy can be saved. First, by virtue of Eq. (20), it is independent on where it is calculated. Secondly, it takes into account the pressure field interactions. This FoM tells us how much the reference configuration was improved but it does not provide information as to how much the reference configuration could have been improved.

EXergy-WASte CoEfFicient When considering a given configuration it would be desirable to evaluate the potential for improvement. This can be achieved by the following exergy-waste coefficient:

$$
E W C:=\frac{\dot{\mathcal{E}}_{\text {rec }}}{\dot{\mathcal{E}} \text { prop }_{\text {REF }}}=\frac{\text { Recoverable Exergy }}{\text { Reference Propulsive Exergy }}
$$

This FoM quantifies how much exergy is flowing out of the control volume; that outflow being recoverable. It gives an indication of how inefficient is the initial configuration. High values indicate high potential for BLI arrangement and equivalently poor usage of the initial available exergy.

The difficulty lies in the definition of the Recoverable Exergy because the exergy potential is destroyed downstream the configuration, see Eqs. $(20 \& 25)$. As a consequence, $\dot{\mathcal{E}}_{\text {rec }}$ depends on where that potential is evaluated and this information should be clearly stated to avoid any confusion. More information will be given in the section III.C.1.

EXERGY-RECOvery COEFFICIENT Finally, having specified the recoverable exergy and the effective exergy saving, it is possible to quantify how efficient was the recovery of the initial potential through an exergyrecovery coefficient:

$$
E R C:=\frac{\dot{\mathcal{E}}_{\text {prop }}^{R E F}-\dot{\mathcal{E}}_{\text {prop }}^{B L I}}{\dot{\mathcal{E}}_{\text {rec }}}=\frac{\text { Exergy Saving }}{\text { Recoverable Exergy }}
$$

High values indicate an efficient BLI arrangement to recover the available exergy. It will be shown in sections III.C.2. and III.C.4. that the difference is due to two mechanisms: destruction by interactions between the two bodies and simple loss.

To conclude, the ESC can be expressed with the two other figures of merit as:

$$
E S C=E R C \times E W C
$$

meaning that a large fuel saving requires a good recovery of a high initial improvement potential.

\section{III.B. Numerical Settings}

\section{III.B.1. Pre-processing - Meshes}

According to the methodology described, three grids are required. First, an isolated fuselage with 172,000 nodes and an isolated nacelle with 533,000 nodes. This ensures the absence of interactions between the two bodies. Secondly, a [fuselage + nacelle] configuration with 694,000 nodes. All grids are structured, axisymmetric, multiblocks and extend about 10 reference length in all three dimensions, see Figs. 15, 16 and 17 in appendix.

\section{III.B.2. Processing - CFD Solver}

Different models can be applied to represent the propulsion system with different level of fidelity. For turbofans, the thrust is mainly produced by the fan itself so that modeling its impact on the flow is a satisfactory approximation. Actuator disks are therefore used in the present analysis. They are relatively average-fidelity as they (only) model the mechanical aspect of the propulsion system. It consists in the addition of a uniform total pressure jump $\Delta P_{i}=P_{i, \text { out }}-P_{i, \text { in }}$ across the discontinuity, see Fig. 7 .

All computations are made using the Onera-elsA code. ${ }^{8}$ This software is here used with a cell-centered finite volume discretization on structured meshes. Time integration is carried out by a backward-Euler 


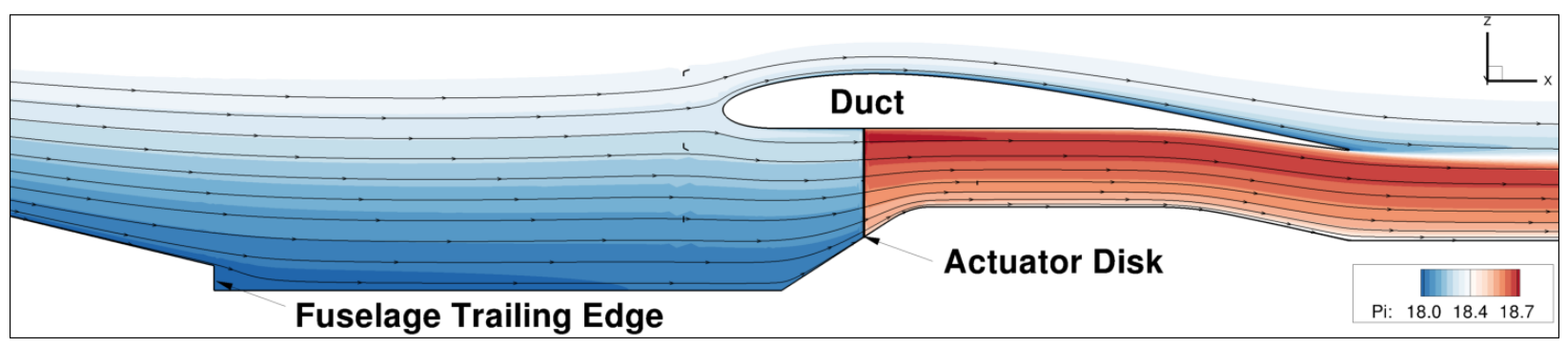

Figure 7. Wake ingestion and actuator disk position. Contours of total pressure and streamlines.

scheme with implicit lower/upper symmetric successive overrelaxation. Spatial discretization is realized through a central Jameson scheme with artificial viscosity $\left(\chi_{2}=1\right.$. and $\left.\chi_{4}=0.032\right)$. All the computations are performed in fully turbulent conditions using the one-equation Spalart-Allmaras model. For the BLI computations, the flow is initialized with a first computation where the nacelle and the fuselage are interacting but the actuator disk is inactive. 60,000 iterations are made to get a 6-order residual reduction, which is considered satisfactory, see Fig. 8. Then 60,000 additional iterations are run to provide again a 6-order residual reduction with the actuator disk active.

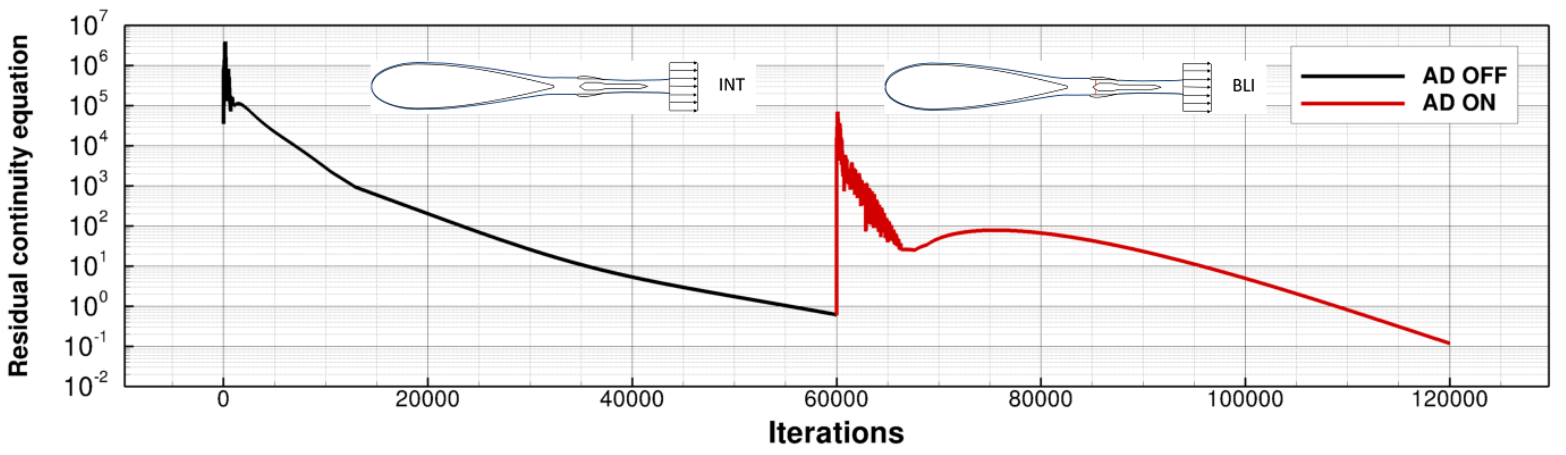

Figure 8. Typical residuals reduction. Black line: actuator disk inactive, red line: actuator disk active.

\section{III.B.3. Post-processing - ffx}

The exergy-based formulation presented in the previous section has been implemented in a Fortran code named far-field exergy, $f f x$. It has been coded as an extension of the in-house code far-field drag $(f f d 72)$ developed by Destarac. ${ }^{10}$ The computation of the average entropy generation rate is made through an eddy viscosity-type formulation as proposed by Alabi. ${ }^{2}$ The formulation and its implementation have been validated through various airframe cases. ${ }^{3}$ It has especially been found that the velocity and temperature gradients of $\dot{\mathcal{A}}_{\phi}$ and $\dot{\mathcal{A}}_{\nabla T}$ are underestimated on coarse meshes.

The outer boundary $S_{O}$ is selected such that the upstream and lateral surfaces are pushed to the mesh boundaries. The downstream surface is a plane perpendicular to freestream velocity $V_{\infty}$ located at fixed $x$-position.

\section{III.B.4. Assumptions and Limits}

The actuator disk boundary condition that has been used does not take into account the flow distortions present in the boundary layer. As a consequence, we implicitly assume that the inlet fan distortion does not modify the fan operating conditions, which is a relatively strong assumption. ${ }^{19}$

Also, the meshes that are used do not contain a great number of cells. As a consequence, the absolute performance coefficients that can be extracted from the flow solutions may be affected. However, as we are interested in comparing two configurations (REF and BLI) that have approximately the same level of inaccuracy, the difference between the coefficients will be meaningful. It is therefore considered that the numerical settings are adapted to the methodology proposed to quantify the physical phenomena underlying the BLI benefit. 


\section{III.C. Wake Ingestion Benefit Investigation}

The investigation considers the 4 following steps:

1. Evaluation of the recoverable exergy and EWC of the reference configuration in III.C.1.

2. Performance comparison of three selected REF/BLI cases in III.C.2.

3. Characterization of the outflows for the case at equilibrium in III.C.3.

4. Quantification of the overall BLI benefit in III.C.4.

A total of 54 computations with various pressure jump across the actuator disk was performed: 27 for the isolated nacelle (REF configuration) and 27 computations for the BLI configuration, as illustrated in Fig. 9. In the following, emphasis will be put on three REF and three BLI cases characterized by an equal net force acting on the configuration which is respectively negative, zero and positive. Case $\mathbf{D}$ when the aircraft is in descent, case $\mathbf{E}$ in equilibrium and case $\mathbf{C}$ when in climb.

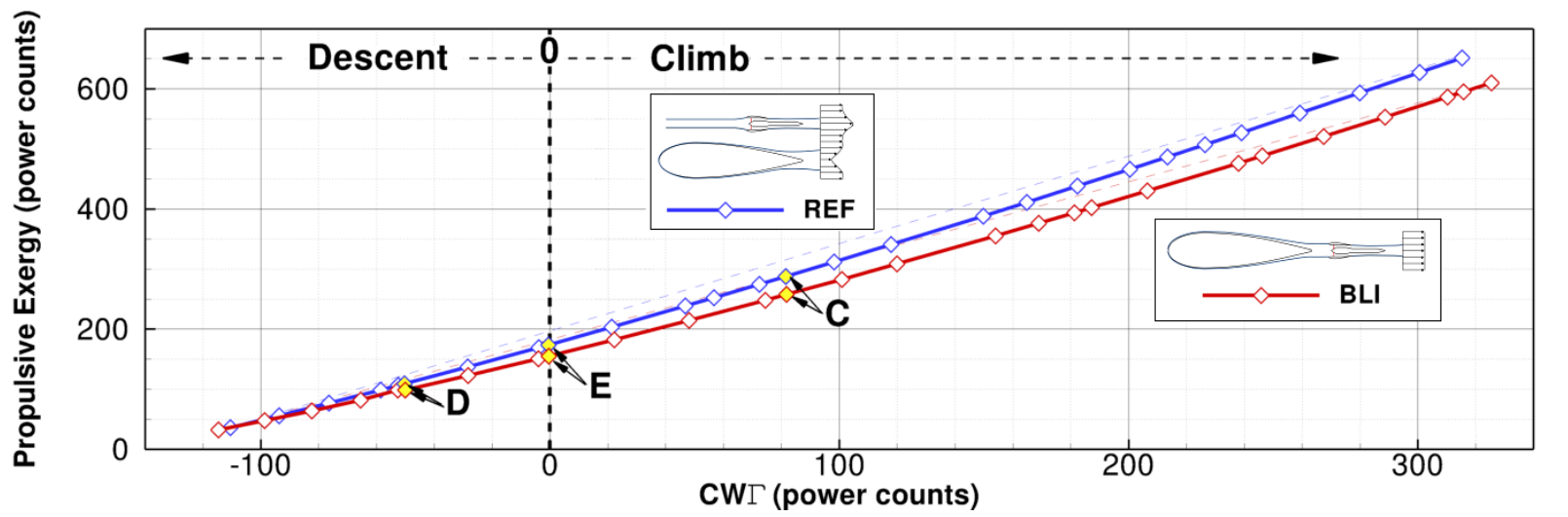

Figure 9. Propulsive exergy versus net force acting on the configuration for the REF configuration (blue) and for the BLI configuration (red).

\section{III.C.1. Nacelle-Jet and Fuselage-Wake Recovery Potential}

According to the methodology described above, the first step consists in determining the recoverable exergy from the reference configuration. The recoverable exergy is defined as the mechanical (and in a lesser extent thermal) exergy flowing out of the control volume. It is crucial to note that both a jet and a wake represent a potential for improvement: the kinetic energy associated with them is independent on whether the flow is locally faster or slower than the freestream velocity: as soon as the flow is not in equilibrium with its environment $\left(u \neq u_{\infty}\right)$, exergy exists. Viscous and thermal anergy will have to be generated to dissipate this work potential. It is therefore important to evaluate how much recovery potential exists. Fig. 10 represents the outflows recoverable exergy destruction when moving the outer boundary downstream a nacelle-jet (a) and the fuselage-wake (b).

For the nacelle (Fig. 10a), the steps-like shape of the curve is due to increasing mesh cell size. The thermal exergy is very negligible, as expected, and the pressure-work and transverse kinetic energy rapidly reduce to zero a few distance downstream, leaving the streamwise kinetic energy as the entire recoverable exergy.

The exergy recovery potential and the EWC are represented in Fig. 11 for a downstream plane located at a given distance downstream of the configuration $\Delta x=0.20$. As regards recoverable exergy, the fuselage contribution is constant ${ }^{\mathrm{h}}$ while the nacelle contribution increases with increasing thrust (and therefore net force). By non-dimensionalizing the total recoverable exergy by the propulsive exergy, we get the EWC (bottom). The curve exhibits a minimum value close to equilibrium which makes the separation between an overall wake $\left(u-u_{\infty}<0\right)$ and an overall jet $\left(u-u_{\infty}>0\right)$. Besides from values extrapolation, pressure field interactions ( $\dot{E}_{p}$ in Fig. 10b) are likely to be the cause of shifting from strict equilibrium.

\footnotetext{
${ }^{\mathrm{h}}$ Around $13 \mathrm{pc}$ which represents around $10 \%$ of its drag.
} 

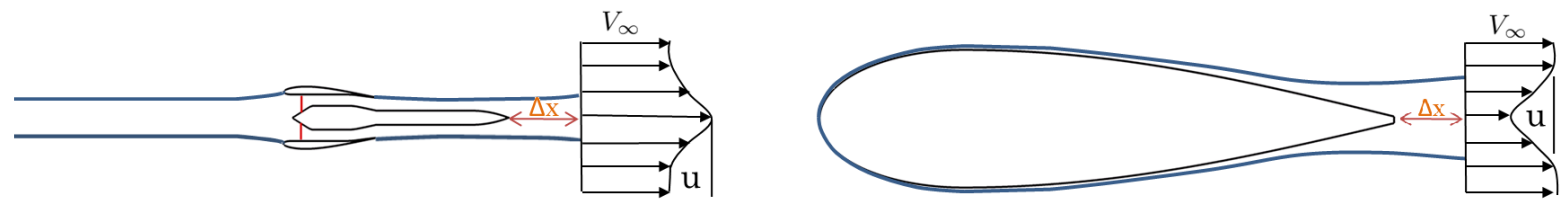

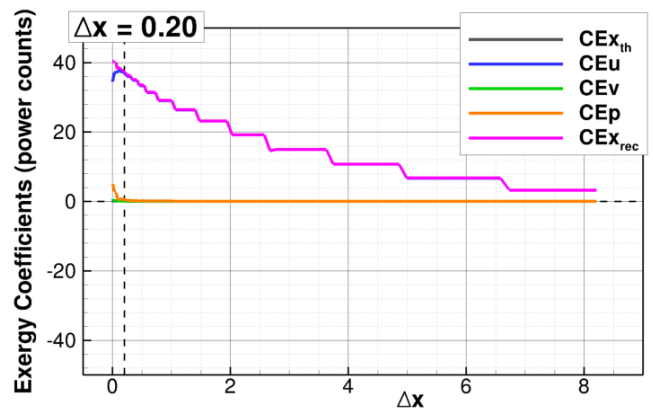

(a) A typical propulsive nacelle-jet.

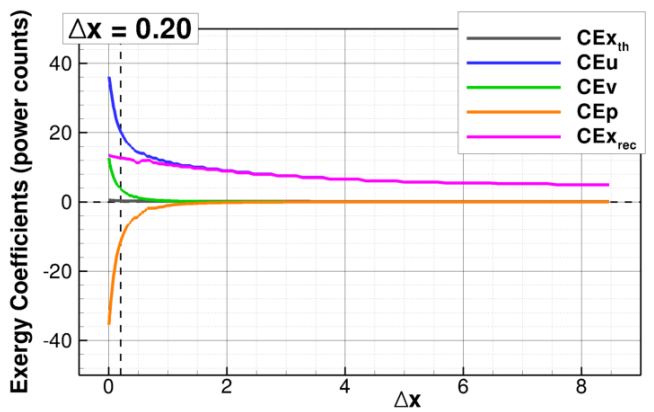

(b) The fuselage-wake.

Figure 10. Outflows recoverable exergy destruction when moving the outer boundary downstream the body.

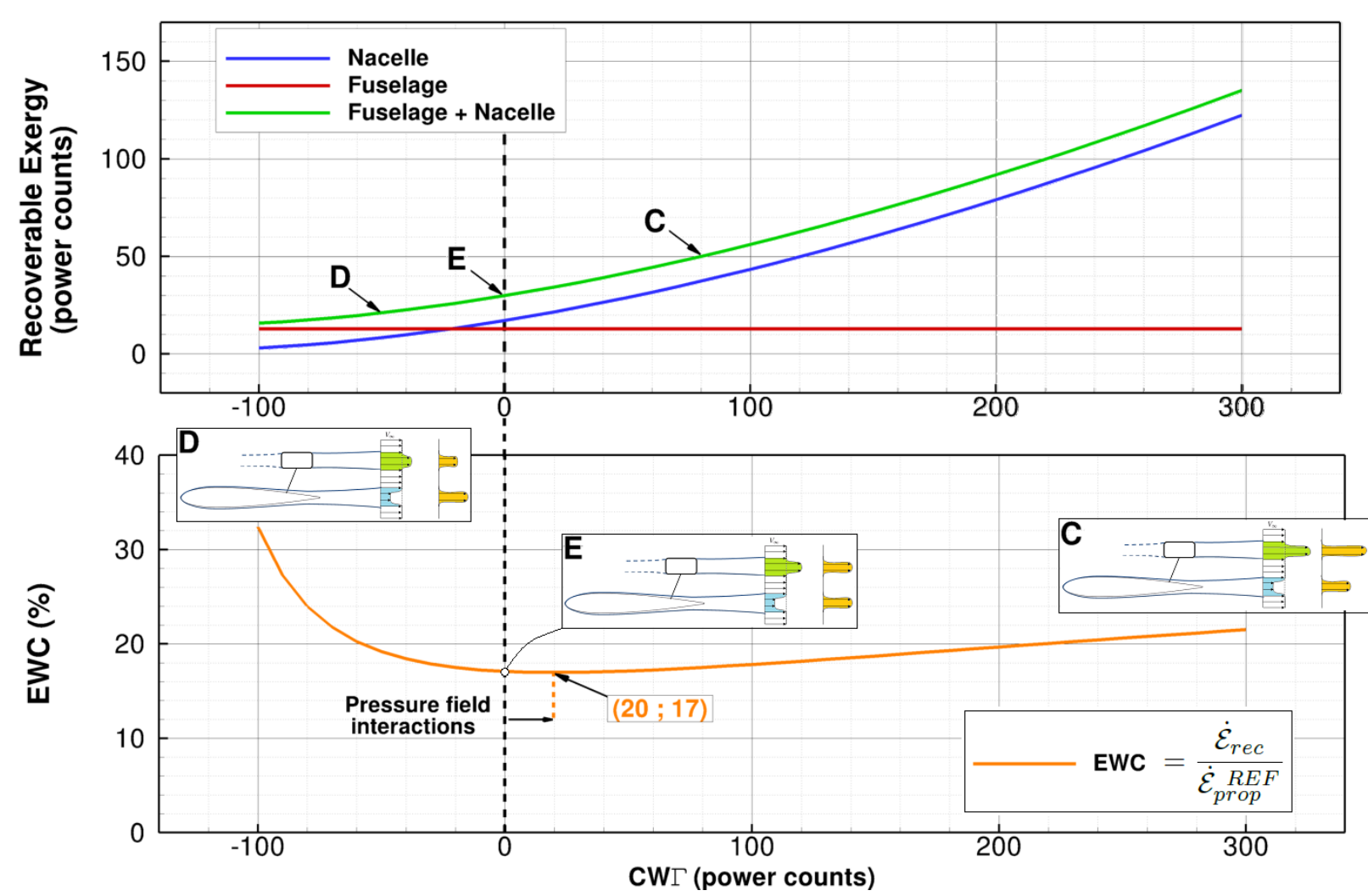

Figure 11. Top: extrapolated recoverable exergy at $\Delta x=0.20$ versus net force acting on the reference configuration. Bottom: EWC versus net force acting on the configuration.

The higher values on the right are associated with the exergy supplied ${ }^{i}$ in excess at greater thrust levels and moderated by higher propulsive exergy. As opposed, the higher values reached at descent are associated with the wake when low levels of thrust are generated and emphasized by low levels of propulsive exergy.

In the following analysis, the nacelle will be located $\Delta x=0.20$ downstream the fuselage.

\footnotetext{
${ }^{\mathrm{i}}$ In the form of kinetic energy.
} 


\section{III.C.2. Far-Field Exergy Comparison}

Now that the recoverable exergy and EWC have been determined, it is possible to compare the BLI configurations to the reference configurations. Qualitatively, the benefit of the BLI configuration is illustrated in Fig. 9 (p. 12) by the vertical distance between the REF curve in blue and the BLI curve in red: for a given net force acting on the configurations, the BLI requires less propulsive exergy.

Quantitatively speaking, Table 1 presents the far-field exergy terms with or without BLI for the three cases mentioned, D, E, and C. The values for REF are the sum of those for the nacelle and for the fuselage, at the same downstream location of the outer boundary $S_{O}(\Delta x=0.20)$. For the BLI cases, the nacelle is located at $\Delta x=0.20$ downstream the fuselage and the outer boundary is located at $\Delta x=0.20$ downstream the nacelle.

\begin{tabular}{cc||rrrr|ccc}
\hline \hline & Configuration & $C W \dot{\Gamma}$ & $C \dot{\mathcal{E}}_{\text {rec }}$ & $C \dot{\mathcal{A}}_{\mathcal{V}}$ & $C \dot{\mathcal{E}}_{\text {prop }}$ & EWC & ERC & ESC \\
\hline \multirow{4}{*}{$\mathrm{D}$} & REF & -50.3 & 20.9 & 138.6 & 109.0 & $19.2 \%$ & - & - \\
& BLI & -50.1 & 2.4 & 146.1 & 98.6 & - & $50.7 \%$ & $9.7 \%$ \\
& BLI - REF & +0.2 & -18.5 & $\mathbf{+ 7 . 5}$ & $\mathbf{- 1 0 . 6}$ & - & - & - \\
\hline \multirow{3}{*}{$\mathbf{E}$} & REF & -0.5 & 30.4 & 144.7 & 174.6 & $17.4 \%$ & - & - \\
& BLI & -0.4 & 3.5 & 152.4 & 155.5 & - & $62.8 \%$ & $10.9 \%$ \\
& BLI - REF & +0.1 & -26.9 & $\mathbf{+ 7 . 7}$ & $\mathbf{- 1 9 . 1}$ & - & - & - \\
\hline \multirow{3}{*}{$\mathbf{C}$} & REF & 81.5 & 50.1 & 156.0 & 287.3 & $17.4 \%$ & - & - \\
& BLI & 81.8 & 10.2 & 164.5 & 258.1 & - & $58.3 \%$ & $10.2 \%$ \\
& BLI - REF & +0.3 & -39.9 & $\mathbf{+ 8 . 5}$ & $\mathbf{- 2 9 . 2}$ & - & - & - \\
\hline \hline
\end{tabular}

Table 1. Far-field exergy terms comparison with or without BLI for a downstream plane located at $\Delta x=0.20$. All exergy coefficients are expressed in power counts.

The following comments can be made:

- From the fist column of results $(C W \dot{\Gamma})$, we can effectively consider that the same force is acting on the two configurations, within 0.3 power counts.

- From the second column we have the recoverable exergy of the reference configuration (Fig 11). Note the great reduction in the recoverable outflows with the BLI configuration. This point will be described in greater details in the following section.

- The third column indicates the (total ${ }^{\mathrm{j}}$ ) anergy generated within the control volume, or equivalently, the exergy destroyed. We observe a non-negligible increase with the BLI configuration meaning that some of the initial recovery potential has been destroyed by the interaction between the two solids. For case $\mathrm{E}$ for example, if this interaction anergy was negligible, the exergy saving would rise from 19.1 to 26.8, thereby improving the ERC from $62.8 \%$ to $\mathrm{ERC}^{*}=88 \%$ and the ESC up to $15.3 \%$.

- The fourth column represents the exergy delivered by the propulsion system and is the sum of the three previous terms, see Eq. (20). We observe a large reduction of the propulsive exergy when in the BLI configuration.

The exergy-waste coefficient (EWC) is around $18 \%$ which is a relatively high figure due to the ingestion of the entire fuselage-wake. The exergy-recovery coefficient (ERC) ranges from $50 \%$ to $60 \%$ meaning that about half of the initial potential was effectively recovered, the rest being lost or destroyed by interaction. This figures yield a global exergy-saving coefficient (ESC) of about $10 \%$.

A rapid estimation of the $\mathrm{EWC}_{B L I}$ can be made from the data available in Table 1. Only $2-4 \%$ of the propulsive exergy would be recoverable at a downstream location of $\Delta x=0.20$ meaning that placing a second nacelle downstream the first one would not make sense. This low figure equivalently signifies that more than $96 \%$ of the propulsive exergy was used wisely.

\footnotetext{
${ }^{\mathrm{j}}$ Mainly viscous anergy.
} 


\section{III.C.3. Outflows Characterization for the Case at Equilibrium}

The BLI benefit having been clearly quantified, it appears that the exergy saving is mainly due to a great reduction of the various recoverable outflows. Therefore Table 2 provides more detailed information about the outflows downstream the configurations for case E.

\begin{tabular}{cc||rrrr|r}
\hline \hline Configuration & Body & $C \dot{E}_{u}$ & $C \dot{E}_{v}$ & $C \dot{E}_{p}$ & $C \dot{\mathcal{E}}_{t h}$ & $C \dot{\mathcal{E}}_{r e c}$ \\
\hline \multirow{4}{*}{ REF } & Fuselage & 20.6 & 3.8 & -11.7 & 0.6 & 13.3 \\
& Nacelle & 16.6 & 0.1 & 0.3 & 0.1 & 17.1 \\
& Nacelle + Fuselage & 37.2 & 3.9 & -11.4 & 0.7 & 30.4 \\
\hline BLI & Nacelle + Fuselage & 3.8 & 0.6 & -1.2 & 0.3 & 3.5 \\
\hline BLI - REF & Nacelle + Fuselage & -33.4 & -3.3 & +10.2 & -0.4 & -26.9 \\
\hline \hline
\end{tabular}

Table 2. Detailed far-field exergy outflows comparison with or without BLI for case $\mathbf{E}$ and a downstream plane located at $\Delta x=0.20$. All exergy coefficients are expressed in power counts.

From the first column $\left(C \dot{E}_{u}\right)$ we can see that the streamwise kinetic energy has nearly vanished downstream of the BLI configuration. Also, the second and third columns indicate that the transverse kinetic energy and boundary pressure-work have been noticeably reduced, being almost negligible for the BLI configuration. The thermal exergy is clearly negligible compared to the mechanical terms.

Except for the streamwise kinetic energy ${ }^{\mathrm{k}}$, the outflows downstream the BLI configuration are comparable to those downstream the isolated nacelle, see lines 2 and 4 . In other words, to the leading order, the BLI arrangement is almost equivalent to having removed the fuselage wake. By doing so, the anergy (entropy ${ }^{18}$ ) that would have been required to bring the velocity perturbation back to equilibrium is avoided: because less exergy is destroyed, less exergy needs to be supplied.

Although, it appears that the overall benefit comes from various source, the largest exergy form that is dealt with in this application is the flow streamwise kinetic energy $\dot{E}_{u}$. Fig. 12 presents the evolution of the streamwise kinetic energy outflow when the plane of the outer boundary $S_{O}$ is moved over the nacelle. By doing so, we scan the configuration and can pinpoint the location of great variations. The thick line represents the isolated nacelle of the reference case, i.e. when the bodies are assumed sufficiently far from each other that we can simply sum the separate terms. The thin and dotted line represents the BLI case.

Qualitatively speaking, from the relative velocity-squared field on top of Fig. 12, one can see that there is very little kinetic energy in the jet of the nacelle, suggesting that this case is close to the ideal one where no exergy, in the form of kinetic energy, is wasted, see Fig. 13.

Quantitatively speaking, comparing the $\dot{E}_{u}$ evolution for the isolated nacelle and the BLI configuration, one can see that:

- For the BLI case, we observe non-zero values $(\geq 40 p c)$ which indicate perturbations generated by the fuselage, i.e. its wake.

- For both cases, the flow is slowed down when approaching the engine inlet: there is a conversion of kinetic energy into pressure energy.

- The dramatic decrease of the kinetic energy around $x=0.0$ is associated with the acceleration of the flow around the duct leading edge. Afterwards, the velocity is increased due to a reduction of the channel area (incompressible flow) around the actuator disk (red line).

- The (total) pressure jump across the actuator disk is then converted into kinetic energy through the engine's exhaust. As a consequence, the axial velocity increases up to the nacelle exhaust and at about half the nacelle length the velocity nearly matches the one of the freestream at which point the kinetic energy exhibits a minimum value.

- The flow is further accelerated in both cases and remains relatively constant for the isolated nacelle. On the other hand, the BLI kinetic energy is rapidly dissipated probably by mixing with the duct wake. At $\Delta x=0.20$, there is a 12.8 pc reduction compared to the reference.

\footnotetext{
${ }^{\mathrm{k}}$ The BLI configuration has a jet kinetic energy which is even lower than the one of the isolated nacelle.
} 


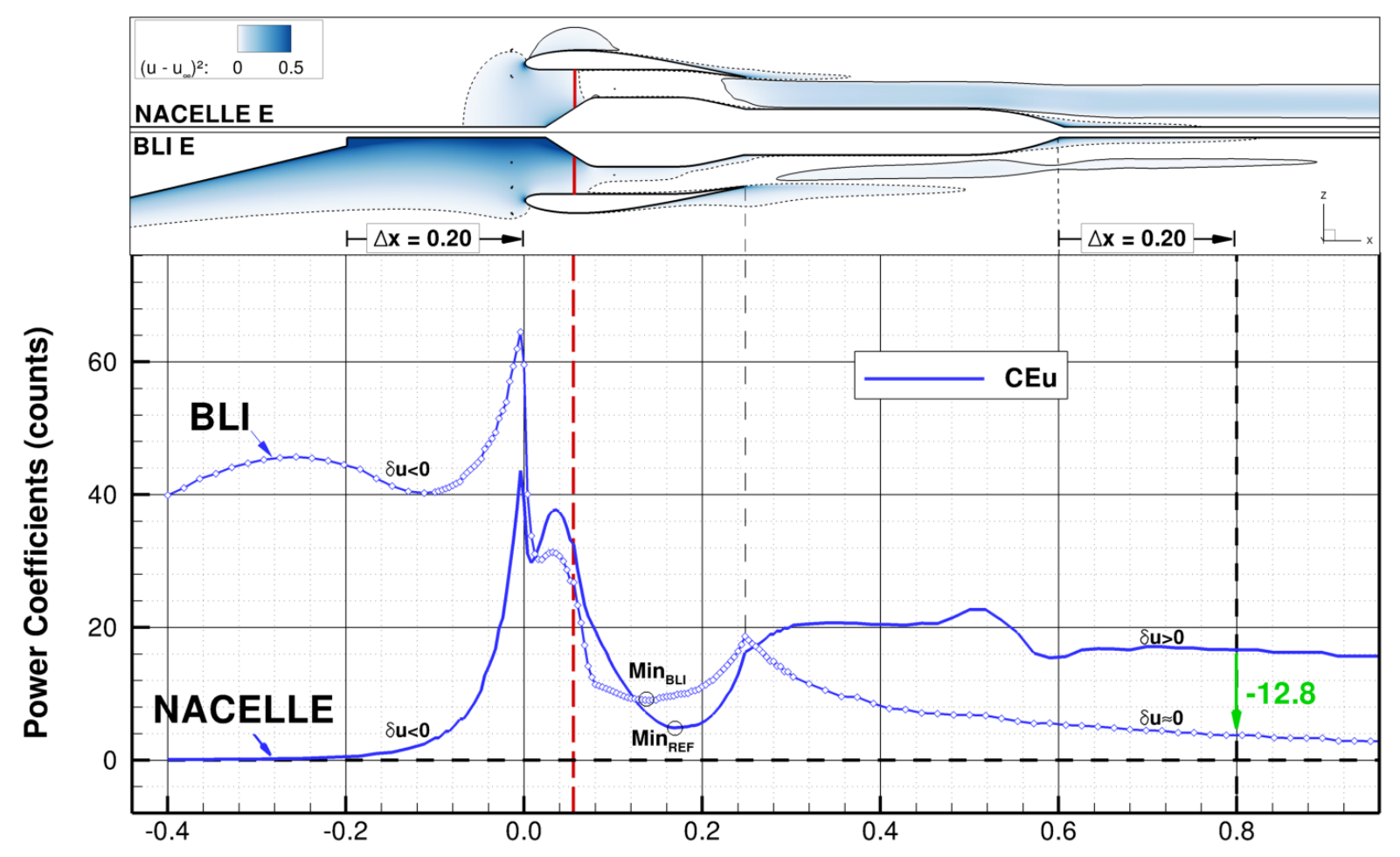

Figure 12. Top: contours of relative velocity squared for the nacelle and BLI when at equilibrium (Case E). Dashed line enclose regions of negative relative axial velocity (deficit) while solid line enclose regions of positive relative axial velocity (excess). Bottom: Comparison of $C \dot{E}_{u}$ with or without wake ingestion. Thick line indicates the reference configuration while the thin and dotted line indicates BLI configurations

\section{Case $\mathrm{E}$}
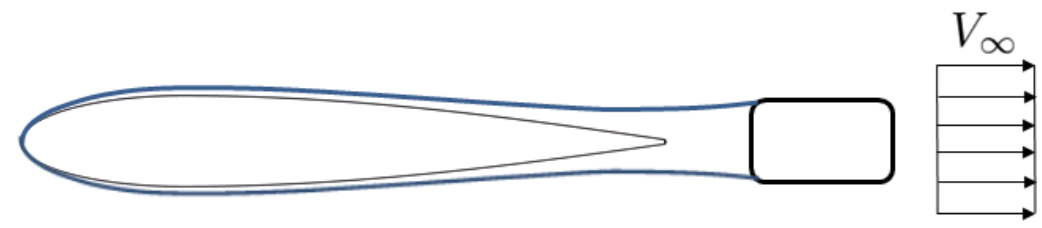

Figure 13. Case E (at equilibrium) is close to the ideal BLI configuration.

Note that the massflow rate of the internal flow (through the engine) is reduced by roughly $12 \%$ which is the reason for lower energy (exergy) supply to the flow.

\section{III.C.4. BLI Benefit Overview}

A summary of the points that have been made in the above sections is provided by Fig. 14 which gives the variation of the three coefficients that quantify the BLI benefit.

The exergy delivered by the propulsive system (top) and the ESC (bottom) are plotted in Fig. 14a. Again, the direct benefit of the BLI configuration can be observed by the vertical distance between the two curves. Note that they nearly cross at $C W \dot{\Gamma}=-100 p c$ : there is an overall negative impact when the propulsive system does not generate great levels of thrust. In other words, the two bodies have a negative impact when placed close from one another: there is an increase in anergy generation (or equivalently exergy destruction).

However, it appears that the BLI arrangement presents a benefit over a wide range of $C W \dot{\Gamma}$ : the ESC ranges from 5 to $11 \%$ at descent, but more importantly exhibits a maximum of $\mathrm{ESC}_{\max }=10.9 \%$ near equilibrium and stays relatively constant for climb cases. As more thrust is generated, the ESC drops down to $9 \%$ for $C W \dot{\Gamma}=300 p c$. As mentioned earlier, this figure of merit is critical as it directly indicates how much fuel could be saved. However, in a design perspective, this coefficient does not tell the designer how much improvement was in fact achievable. 


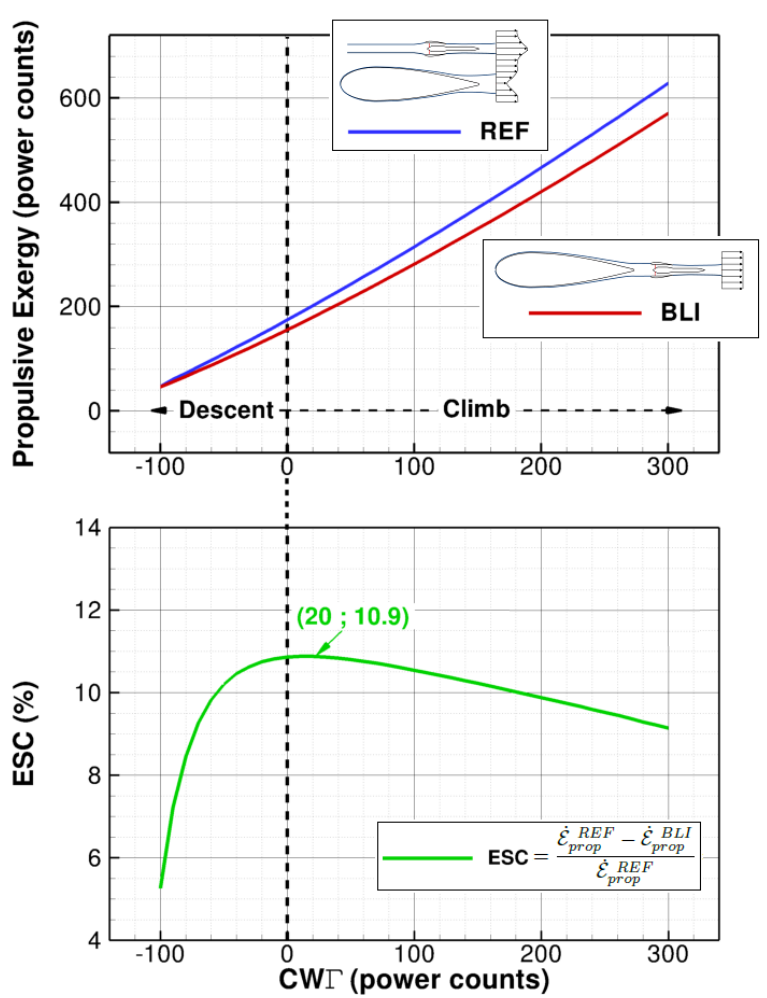

(a) Top: total power coefficients for reference configuration (blue) and BLI configuration (red) versus change in energy height. Bottom: ESC versus change in energy height.

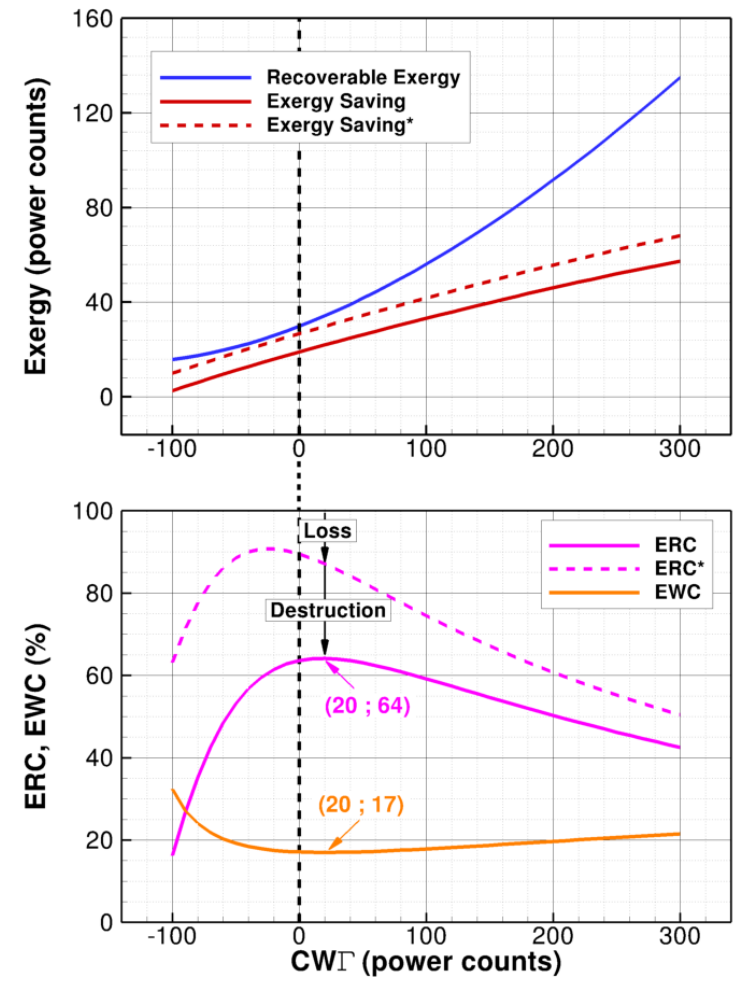

(b) Top: recoverable exergy (blue), actual and possible exergy saving (red) versus change in energy height. Bottom: EWC, ERC and ERC* versus change in energy height.

Figure 14. BLI performance coefficients summary

Fig. 14b gives more information about how much improvement was possible and how efficient was the recovery of the initial potential. Top figure recalls the recoverable exergy (blue), which, divided by the propulsive exergy, yields the EWC (orange). This coefficient exhibits a minimum $\mathrm{EWC}_{\min }=17 \%$ near equilibrium. As we have seen, the higher values on the right are associated with the exergy supplied ${ }^{1}$ in excess at greater thrust levels and moderated by higher propulsive exergy. As opposed, the higher values reached at descent are associated with the wake when low levels of thrust are generated and magnified by low levels of propulsive exergy.

Top figure also gives the exergy effectively recovered (solid red) and the exergy that could have been recovered in the absence of interaction anergy (dashed red). Bottom figure shows that ERC (solid purple) exhibits a maximum of $\mathrm{ERC}_{\max }=64 \%$ at $C W \dot{\Gamma}=20 \mathrm{pc}$. There are two identified reasons for not recovering $100 \%$ of the recoverable exergy. First, part of it has been destroyed by interaction anergy, i.e. the additional exergy destroyed due to the interaction of the two bodies, see ERC* (dashed purple). The second reason is the loss of that potential which could suggest that improvements are still possible. In descent, the destruction is the most important cause while at climb the loss appears predominant.

Interestingly, the maximum exergy saving (ESC) occurs when the improvement potential (EWC) is the lowest but its recovery (ERC) is the highest. From an exergy standpoint, less exergy has to be supplied with the BLI configuration because less exergy is destroyed to bring the wake/jet back to thermodynamic equilibrium with its environment.

\footnotetext{
${ }^{1}$ In the form of kinetic energy.
} 


\section{Conclusions \& Perspectives}

First, an exergy-based formulation has been derived combining a momentum balance along with a first and second laws analysis. The output of the derivation is a balance between the exergy supplied by the propulsion system and its (partial) destruction within the control volume. Relation is made with the aircraft mechanical equilibrium and the various outflows that could be recovered. Additionally, the flow phenomena that directly influence flight power requirements are identified. The choice of exergy is motivated by its ability to provide a consistent framework to design complex aerospace systems.

Secondly, the formulation was applied to an academic BLI configuration to investigate the benefit of wake ingestion with high fidelity (CFD) tools and realistic engine conditions. An actuator disk boundary condition was applied to model the effects on the flow of a propeller. In an exergy point of view, the underlying principle of the BLI principle is the recovery of the streamwise kinetic energy of the preceding body, the fuselage. For the present configuration, an ESC in the range of $10 \%-11 \%$ is found for a complete wake ingestion using a simple actuator disk to model a propeller. An exergy-waste coefficient (EWC) has been introduced to determine the potential for improvement of the reference configuration. Loss and destruction of the recoverable exergy due to interaction have been quantified.

Finally, the future work will focus on the analysis of the configurations at greater Mach numbers where the propulsion system may take advantage of the thermal exergy of the fuselage-wake. Perspectives also include a more realistic boundary-layer ingestion case consisting of a simplified geometry representing a blended wing-body configuration with a BLI propulsion system on top. This test case could also feature heat transfer at the aircraft surface.

\section{Acknowledgments}

The authors thank the French National Research Agency (Carnot project) who supported the work and R. Grenon (ONERA) for providing the grids.

\section{References}

${ }^{1}$ Abeyounis, W. K., Patterson, Jr., J. C., Stough, III, H. P., Wunschel, Lt. Col., A. J., and Curran, P. D., "Wingtip Vortex Turbine Investigation for Vortex Energy Recovery," SAE Aerotech '90, 1990 SAE Aerospace Technology Conference \& Exposition, 1-4 October, 1990.

${ }^{2}$ Alabi, K., Ladeinde, F., von Spakosvky, M., Moorhouse, D. and Camberos, J. A. "The Use of the $2^{\text {nd }}$ Law as a Potential Design Tool for Aircraft Air Frame Subsystems", Int. J. of Thermodynamics, 9 (4), pp. 1-14, 2006.

${ }^{3}$ Arntz, A., Atinault, O., Destarac, D. and Merlen, A. "Numerical Airframe Aerodynamic Performance Prediction: An Exergy Point of View", 49 ${ }^{\text {th }}$ International Symposium of Applied Aerodynamics, AAAF, Lille, France, 24-26 March, 2014.

${ }^{4}$ Atinault, O., Carrier, G., Grenon, R., Verbecke, C. and Viscat, P. "Numerical and Experimental Aerodynamic Investigations of Boundary Layer Ingestion for Improving Propulsion Efficiency of Future Air Transport", AIAA 2013-2406, 31 ${ }^{\text {st }}$ AIAA Applied Aerodynamics Conference, San Diego, CA, 2013.

${ }^{5}$ Bejan, A. "Entropy Generation Minimization: The Method of Thermodynamic Optimization of Finite-Size Systems and Finite-Time Processes", CRC Press Inc., 1996.

${ }^{6}$ Bejan, A. "Fundamentals of Exergy Analysis, Entropy Generation Minimization, and the Generation of Flow Architecture", Int. J. Energy Res., 26, pp. 545-565, 2002.

${ }^{7}$ Betz, A. "Introduction to the Theory of Flow Machines", Pergamon Press, 1996.

${ }^{8}$ Cambier, L., Heib, S. and Plot, S. "The Onera elsA CFD Software: Input from Research and Feedback from Industry", Mechanics $\mathcal{E}$ Industry, 14 (3), pp. 159-174, 2013.

${ }^{9}$ Cengel, Y. A. and Boles, M. A. "Thermodynamics: An Engineering Approach", $5^{\text {th }}$ Ed., McGraw-Hill Higher Education, Boston, MA, 2006.

${ }^{10}$ Destarac, D. "Far-field / Near-field Drag Balance and Applications of Drag Extraction in CFD", CFD-based Aircraft Drag Prediction and Reduction Conference, Hampton, VA, 2003.

${ }^{11}$ Dincer, I. and Rosen, M. A. "Exergy, Energy, Environment and Sustainable Development", Elsevier Science Ltd., 2007.

${ }^{12}$ Drela, M. "Power Balance in Aerodynamic Flows", AIAA Journal, 47 (7), pp. 1761-1771, 2009.

${ }^{13}$ Giles, M. B. and Cummings, R. M. "Wake Integration for Three-Dimensional Flowfield Computations: Theoretical Development", Journal of Aircraft, 36 (2), pp. 357-365, 1999.

${ }^{14}$ Keenan, J. H. "Availability and irreversibility in thermodynamics", British Journal of Applied Physics, 2 (7), pp. 183-192, 1951.

${ }^{15}$ Kless, J. E., Aftosmis, M. J., Ning, S. A., and Nemec, M., "Inviscid Analysis of Extended-Formation Flight," AIAA Journal, Vol. 51, No. 7, 2013, pp. 1703-1715. doi: 10.2514/1.J052224

${ }^{16} \mathrm{Li}$, H., Stewart, J. and Figliola, R. S. "Exergy Based Design Methodology for Airfoil Shape Optimization and Wing analysis", $25^{\text {th }}$ Congress of the International Council of the Aeronautical Sciences, 2006. 
${ }^{17}$ Oswatitsch, K. "Gas Dynamics", Academic Press Inc., New-York, 1956.

${ }^{18}$ Pandya, S. A., Huand, A., Espitia, A., and Uranga, A., "Computational Assessment of the Boundary Layer Ingesting Nacelle Design of the D8 Aircraft," 52 ${ }^{\text {th }}$ AIAA Aerospace Sciences Meeting, National Harbor, MD, 13-17 January 2014.

${ }^{19}$ Plas, A. "Performance of Boundary Layer Ingesting Propulsion System", MSc Thesis, Massachusetts Institute of Technology, 2006.

${ }^{20}$ Rant, Z. "Exergie, ein neues Wort für "technische Arbeitsfähigkeit"”, Forsch. Ing. Wes., 22 (1), pp. 36-37, 1956.

${ }^{21}$ Riggins, D. W., Moorhouse, D. J. and Camberos, J. A. "Characterization of Aerospace Vehicle Performance and Mission Analysis Using Thermodynamic Availability", Journal of Aircraft, 47 (3), pp. 904-916, 2010.

${ }^{22}$ Roth, B. "Work Potential Perspective of Engine Component Performance", Journal of Propulsion and Power, 18 (6), pp. 1183-1190, 2002.

${ }^{23}$ Sato, S. "The Power Balance Method for Aerodynamic Performance", PhD Thesis, Massachusetts Institute of Technology, 2012.

${ }^{24}$ Sciubba, E. and Wall, G. "A brief Commented History of Exergy From the Beginnings to 2004", Int. J. of Thermodynamics, 10 (1), pp. 1-26, 2007.

${ }^{25}$ Smith, L. H. "Wake Ingestion Propulsion Benefit", Journal of Propulsion and Power, 9 (1), pp. 74-82, 1993.

${ }^{26}$ Uranga, A., Drela, M., Greitzer, E. M., Titchener, N. A., Lieu, M. K., Siu, N. M., Huang, A. C., Gatlin, G. M., and Hannon, J. A., "Preliminary Experimental Assessment of the Boundary Layer Ingestion Benefit for the D8 Aircraft," $52^{\text {th }}$ AIAA Aerospace Sciences Meeting, National Harbor, MD, 13-17 January 2014. 


\section{Appendix}
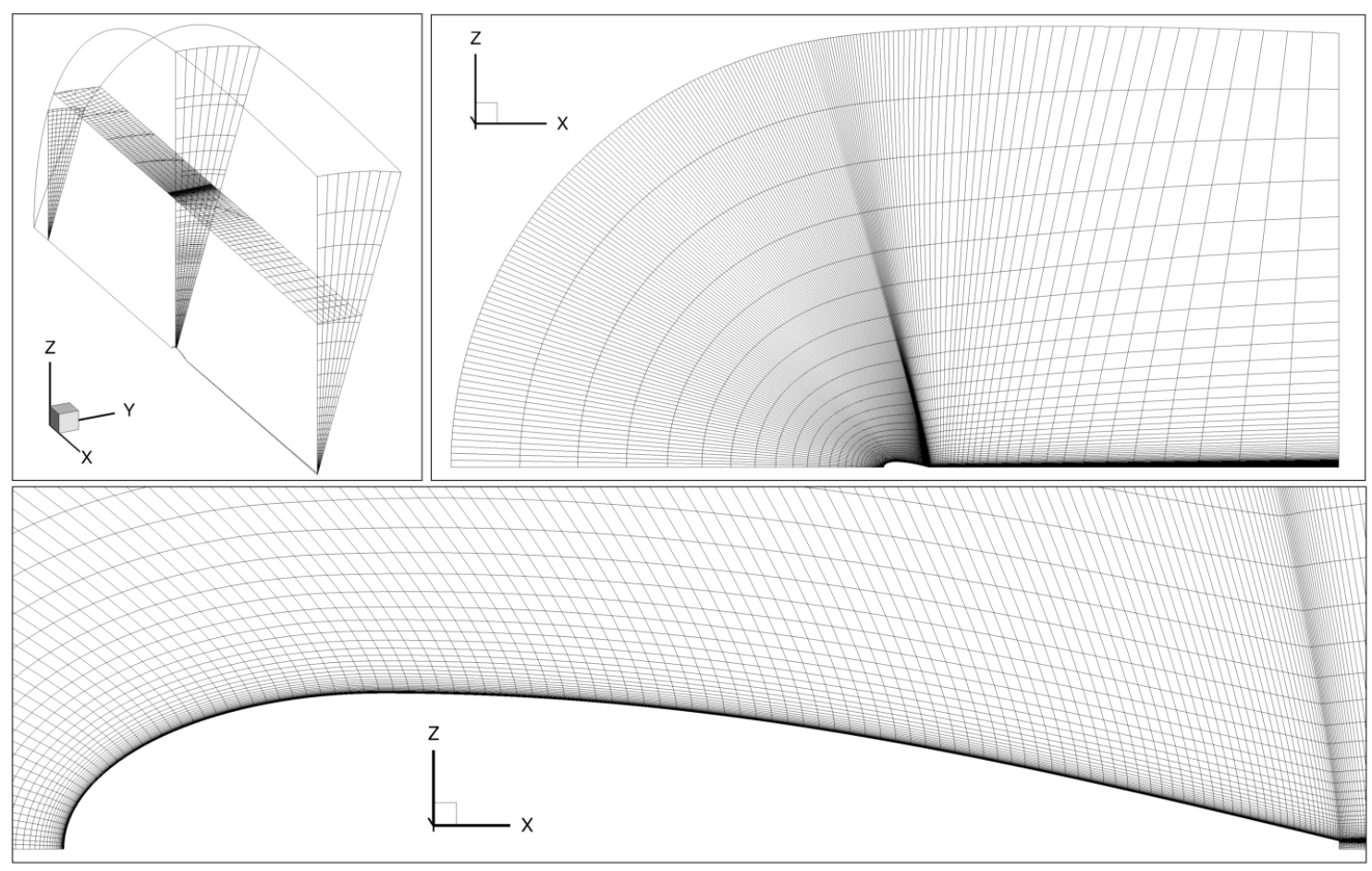

Figure 15. Isolated Fuselage Grid. Top left: 3D view, top right: X-Z Plane view, bottom: zoom on fuselage.

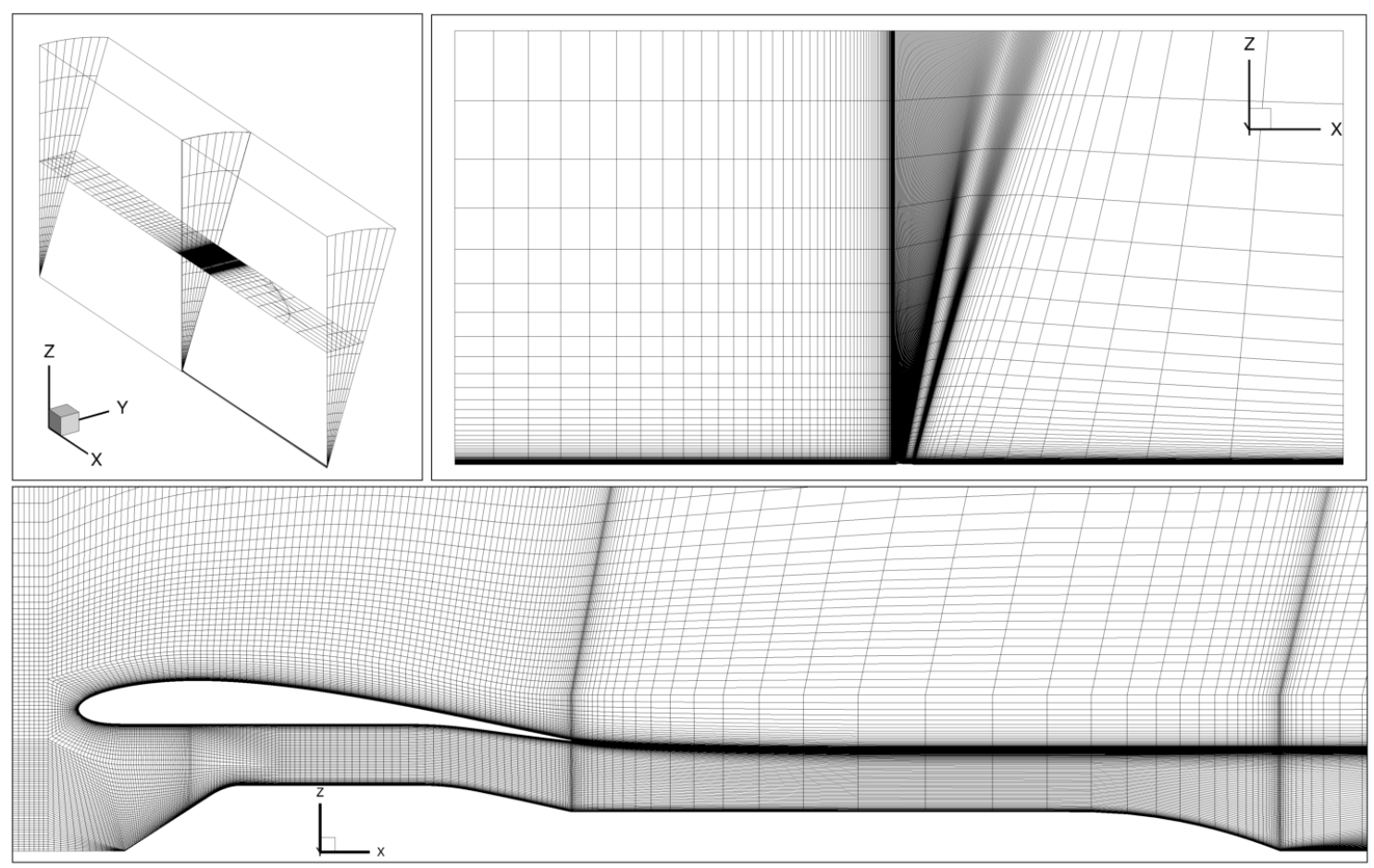

Figure 16. Isolated Nacelle Grid Description. Top left: 3D view, top right: X-Z Plane view, bottom: zoom on nacelle. 


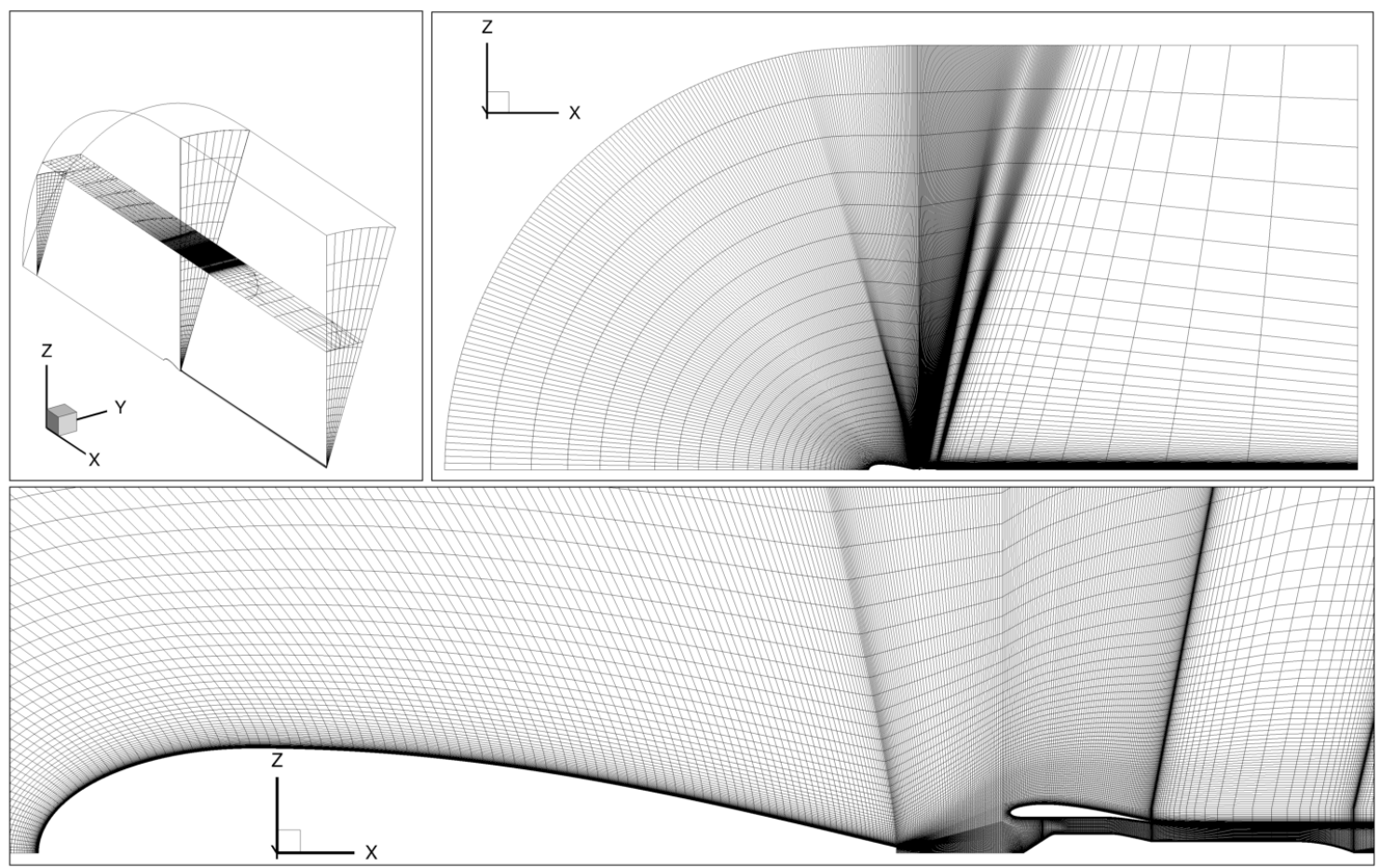

Figure 17. Fuselage + Nacelle Grid Description. Top left: 3D view, top right: X-Z Plane view, bottom: zoom on fuselage + nacelle. 